\title{
Dynamic Analysis of Multilayers Based MEMS Resonators
}

\author{
Hassen M. Ouakad, ${ }^{1}$ Abdulrahman M. Alofi, ${ }^{1,2}$ and Ali H. Nayfeh ${ }^{3,4}$ \\ ${ }^{1}$ Department of Mechanical Engineering, King Fahd University of Petroleum and Minerals, Dhahran 31261, Saudi Arabia \\ ${ }^{2}$ Mechanical Engineering Department, University of Maryland, College Park, MD 20742, USA \\ ${ }^{3}$ Department of Engineering Science and Mechanics, Virginia Polytechnic Institute and State University, Blacksburg, VA 24061, USA \\ ${ }^{4}$ Department of Mechanical Engineering, University of Jordan, Amman, Jordan
}

Correspondence should be addressed to Hassen M. Ouakad; houakad@kfupm.edu.sa

Received 28 September 2016; Revised 20 November 2016; Accepted 30 November 2016; Published 5 January 2017

Academic Editor: Angelo Di Egidio

Copyright (C) 2017 Hassen M. Ouakad et al. This is an open access article distributed under the Creative Commons Attribution License, which permits unrestricted use, distribution, and reproduction in any medium, provided the original work is properly cited.

\begin{abstract}
The dynamic behavior of a microelectromechanical system (MEMS) parallel and electrically coupled double-layers (microbeams) based resonator is investigated. Two numerical methods were used to solve the dynamical problem: the reduced-order modeling (ROM) and the perturbation method. The ROM was derived using the so-called Galerkin expansion with considering the linear undamped mode shapes of straight beam as the basis functions. The perturbation method was generated using the method of multiple scales by direct attack of the equations of motion. Dynamic analyses, assuming the above two numerical methods were performed, and a comparison of the results showed good agreement. Finally, a parametric study was performed using the perturbation on different parameters and the results revealed different interesting features, which hopefully can be useful for some MEMS based applications.
\end{abstract}

\section{Introduction}

Microelectromechanical systems (MEMS) were mostly developed during the industrial revolution in the late of 20th century [1]. From that moment, scientists have continuously investigated the potential of these devices to apply them for different applications. Nowadays, their existence became indispensable in many fields $[2,3]$ and the continuous development hopefully will lead to more importance in future.

When dealing with MEMS based devices, interesting dynamical behaviors can be arising due to the nonlinearity of those devices [4-6]. Among them is their hysteresis (softening and hardening) behavior during the resonance phenomenon. The hardening effect shifts the linear resonance profile to the right and hence produces higher frequencies for the higher amplitude values [7]. A main source of this effect for the clamped-clamped microbeam is the mid-plane stretching. On the other hand, the opposite can be said for the softening effect, which depends on the quadratic nonlinearity effect that may arise from the nonlinear electrical forces [8].

Solving nonlinear dynamical behaviors for MEMS devices is fundamental, since it helps in accurately characterizing and designing them to obtain the desired features quickly and effectively. However, the resolution could be sometime cumbersome and many researchers have struggled to find an effective way to tackle this problem [9-12]. The key techniques in solving such nonlinear problems involve reducing the order of the partial differential governing equation, which sometime is difficult to be solved, into ordinary differential equations, which are much easier to deal with. Different approaches are suggested to solve equation; the reducedorder modeling (ROM) and the perturbation analysis are among those approaches.

In recent years, many researchers extensively used reduced-order modeling (ROM) techniques to obtain the structural behavior of MEMS microstructures [13, 14]. It became nowadays among the most common numerical methods used in the MEMS community. In 2003, Younis et al. [15] presented a new ROM (macromodel) to solve for the nonlinear partial differential equation. They found that their method is more attractive than the finite element method in terms of accuracy and cost. Nayfeh et al. [16] reviewed the work of ROM in MEMS devices. They classified the ROM into two main categories: node and domain methods. 


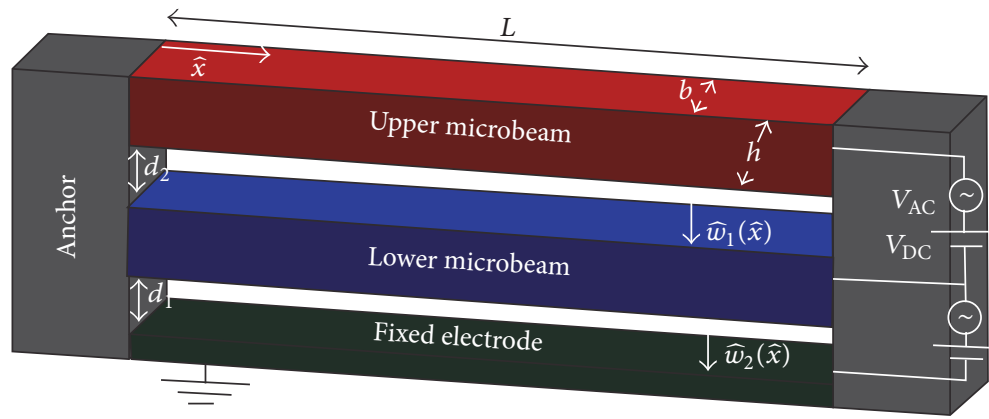

FIGURE 1: 3D schematic of a MEMS double-layers MEMS based resonator configuration.

Perturbation method is also another approach used in the investigation. Turner and Andrews [17] used the perturbation method to obtain an approximation for the nonlinear resonance frequency of a microbeam. They modeled the problem by using a spring mass model, and they included a cubic restoring force to represent the mid-plane stretching. Younis and Nayfeh [6] used the method of multiple scales, as a perturbation method, in a model that accounted for the electrical loads (both DC and AC) and for the mid-plane stretching for a clamped-clamped single-microbeam resonator.

In a previous study [18], we examined the static behavior of a double-layers based clamped-clamped microbeams MEMS based actuator configuration. This work considers the nonlinear dynamical behaviors for this MEMS structure by using the ROM and the perturbation method. This is due to the different features this device has as it is compared with the single microbeam. For example, the double-microbeam configuration proved its capability in reducing the actuation and the pull-in voltage, and nowadays there are many available investigations that used this type of configuration [19-23].

We will start our present numerical investigation by discussing first the problem formulation. Then, the reducedorder model for double-layers MEMS based resonator will be derived and followed by the analysis for the perturbation method. Afterward, a comparison will be carried out for each method to validate both approaches. Finally, a parametric study will be done to investigate some dynamical features for the microactuator.

\section{Problem Formulation}

The proposed model is shown in Figure 1. The suggested MEMS device is mainly made of two parallel electrically actuated microbeams. Both microbeams are clamped-clamped and actuated by both a static DC voltage (of amplitude $V_{\mathrm{DC}}$ ) and a harmonic AC bias (of amplitude $V_{\mathrm{AC}}$ ).

The governing equations of motion for upper and lower microbeams of the MEMS multilayers based resonator are given, respectively, as follows:

$$
\begin{gathered}
E I \frac{\partial^{4} \widehat{w}_{1}}{\partial \widehat{x}^{4}}+\rho b h \frac{\partial^{2} \widehat{w}_{1}}{\partial \hat{t}^{2}}+\widehat{c} \frac{\partial \widehat{w}_{1}}{\partial \hat{t}} \\
=\frac{E A}{2 L} \int_{0}^{L}\left(\frac{\partial \widehat{w}_{1}}{\partial \widehat{x}}\right)^{2} d \widehat{x} \frac{\partial^{2} \widehat{w}_{1}}{\partial \widehat{x}^{2}}
\end{gathered}
$$

$$
\begin{array}{r}
+\frac{\varepsilon_{0} b\left(V_{\mathrm{DC}}+V_{\mathrm{AC}} \cos (\widehat{\Omega} \hat{t})\right)^{2}}{2\left(d_{1}-\widehat{w}_{1}\right)^{2}} \\
-\frac{\varepsilon_{0} b\left(V_{\mathrm{DC}}+V_{\mathrm{AC}} \cos (\widehat{\Omega} \hat{t})\right)^{2}}{2\left(d_{2}+\widehat{w}_{1}-\widehat{w}_{2}\right)^{2}}, \\
E I \frac{\partial^{4} \widehat{w}_{2}}{\partial \widehat{x}^{4}}+\rho b h \frac{\partial^{2} \widehat{w}_{2}}{\partial \hat{t}^{2}}+\widehat{c} \frac{\partial \widehat{w}_{2}}{\partial \widehat{t}} \\
=\frac{E A}{2 L} \int_{0}^{L}\left(\frac{\partial \widehat{w}_{2}}{\partial \widehat{x}}\right)^{2} d \widehat{x} \frac{\partial^{2} \widehat{w}_{2}}{\partial \widehat{x}^{2}} \\
+\frac{\varepsilon_{0} b\left(V_{\mathrm{DC}}+V_{\mathrm{AC}} \cos \left(\widehat{\Omega}_{\hat{t}}\right)\right)^{2}}{2\left(d_{2}+\widehat{w}_{1}-\widehat{w}_{2}\right)^{2}}
\end{array}
$$

and the associated clamped-clamped boundary conditions are

$$
\begin{aligned}
\widehat{w}_{1}(0, \hat{t}) & =0, \\
\widehat{w}_{1}(L, \widehat{t}) & =0, \\
\frac{\partial \widehat{w}_{1}}{\partial \widehat{x}}(0, \hat{t}) & =0, \\
\frac{\partial \widehat{w}_{1}}{\partial \widehat{x}}(L, \widehat{t}) & =0, \\
\widehat{w}_{2}(0, \widehat{t}) & =0, \\
\widehat{w}_{2} & (L, \widehat{t})=0, \\
\frac{\partial \widehat{w}_{2}}{\partial \widehat{x}}(0, \widehat{t}) & =0, \\
\frac{\partial \widehat{w}_{2}}{\partial \widehat{x}}(L, \widehat{t}) & =0 .
\end{aligned}
$$

The functions $\widehat{w}_{1}(\hat{x}, \widehat{t})$ and $\widehat{w}_{2}(\hat{x}, \widehat{t})$ are, respectively, the inplane deflections of the upper and lower microbeam, respectively. For convenience, the equation of the system will be 
normalized while considering the following nondimensional parameters:

$$
\begin{aligned}
w_{1} & =\frac{\widehat{w}_{1}}{d_{1}}, \\
w_{2} & =\frac{\widehat{w}_{2}}{d_{1}}, \\
x & =\frac{\widehat{x}}{L}, \\
t & =\frac{\widehat{t}}{T},
\end{aligned}
$$

where $T$ is a time scale parameter and is chosen to be $\sqrt{\rho b h L^{4} / E I}$.

Therefore, substituting (4) into (1)-(3) will give

$$
\begin{aligned}
& \frac{\partial^{4} w_{1}}{\partial x^{4}}+\frac{\partial^{2} w_{1}}{\partial t^{2}}+c \frac{\partial w_{1}}{\partial t} \\
& =\alpha_{3} \Gamma_{1} \frac{\partial^{2} w_{1}}{\partial x^{2}}+\frac{\alpha_{4}\left(V_{\mathrm{DC}}+V_{\mathrm{AC}} \cos (\Omega t)\right)^{2}}{\left(1-w_{1}\right)^{2}} \\
& -\frac{\alpha_{4}\left(V_{\mathrm{DC}}+V_{\mathrm{AC}} \cos (\Omega t)\right)^{2}}{\left(d_{2} / d_{1}+w_{1}-w_{2}\right)^{2}}, \\
& \frac{\partial^{4} w_{2}}{\partial x^{4}}+\frac{\partial^{2} w_{2}}{\partial t^{2}}+c \frac{\partial w_{2}}{\partial t} \\
& =\alpha_{3} \Gamma_{2} \frac{\partial^{2} w_{2}}{\partial x^{2}}+\frac{\alpha_{4}\left(V_{\mathrm{DC}}+V_{\mathrm{AC}} \cos (\Omega t)\right)^{2}}{\left(d_{2} / d_{1}+w_{1}-w_{2}\right)^{2}}, \\
& w_{1}(0, t)=0 \text {, } \\
& w_{1}(1, t)=0 \text {, } \\
& \frac{\partial w_{1}}{\partial x}(0, t)=0, \\
& \frac{\partial w_{1}}{\partial x}(1, t)=0, \\
& w_{2}(0, t)=0 \text {, } \\
& w_{2}(1, t)=0 \text {, } \\
& \frac{\partial w_{2}}{\partial x}(0, t)=0, \\
& \frac{\partial w_{2}}{\partial x}(1, t)=0,
\end{aligned}
$$

where the nondimensional parameters defined in (5)-(6) are given as follows:

$$
\begin{aligned}
& \alpha_{1}=6\left(\frac{D}{h}\right)^{2} ; \\
& \alpha_{2}=\frac{6 \varepsilon_{0} L^{4}}{E h^{3} D^{3}}
\end{aligned}
$$

$$
\begin{aligned}
\alpha_{3} & =6\left(\frac{d_{1}}{h}\right)^{2} ; \\
\alpha_{4} & =\frac{6 \varepsilon_{0} L^{4}}{E h^{3} d_{1}^{3}} ; \\
c & =\frac{\widehat{c} L^{4}}{T E I} ; \\
\Gamma & =\int_{0}^{1}\left(\frac{\partial w}{\partial x}\right)^{2} d x ; \\
\Gamma_{i} & =\int_{0}^{1}\left(\frac{\partial w_{i}}{\partial x}\right)^{2} d x .
\end{aligned}
$$

\section{Reduced-Order Modeling (ROM)}

Reduced-order modeling (ROM) for the above described nonlinear problem can be obtained by discretizing (5) and (6) using the Galerkin expansion. Therefore, the deflection for the lower and upper microbeams will have the following forms, respectively:

$$
\begin{aligned}
& w_{1}(x, t)=\sum_{i=1}^{N} p_{i}(t) \phi_{i}(x), \\
& w_{2}(x, t)=\sum_{i=1}^{N} q_{i}(t) \phi_{i}(x),
\end{aligned}
$$

where $\phi_{i}(x)$ are the linear undamped mode shapes of a clamped-clamped microbeam, which are orthogonal. After that, (5) will be multiplied by $\left(1-w_{1}\right)^{2}\left(d_{2} / d_{1}-w_{1}-w_{2}\right)^{2}$ and (6) will be multiplied by $\left(d_{2} / d_{1}-w_{1}-w_{2}\right)^{2}$ since this will help in reducing the computational cost. Next, the outcome will be multiplied by $\phi_{j}(x)$ and then integrated from $x=0$ to $x=1$ to give the following ROM equations:

$$
\begin{aligned}
& \int_{x=0}^{1} \phi_{j}(x)\left(1-\sum_{i=1}^{N} p_{i}(t) \phi_{i}(x)\right)^{2} *\left(\frac{d_{2}}{d_{1}}\right. \\
& \left.+\sum_{i=1}^{N} p_{i}(t) \phi_{i}(x)-\sum_{i=1}^{N} q_{i}(t) \phi_{i}(x)\right)^{2} \\
& *\left(\sum_{i=1}^{N} p_{i}(t) \phi_{i}^{i v}(x)+\sum_{i=1}^{N} \ddot{p}_{i}(t) \phi_{i}(x)\right. \\
& \left.+c \sum_{i=1}^{N} \dot{p}_{i}(t) \phi_{i}(x)\right) d x=\int_{x=0}^{1} \phi_{j}(x) \\
& \quad\left(\left(1-\sum_{i=1}^{N} p_{i}(t) \phi_{i}(x)\right)^{2}\right. \\
& \quad\left(\frac{d_{2}}{d_{1}}+\sum_{i=1}^{N} p_{i}(t) \phi_{i}(x)-\sum_{i=1}^{N} q_{i}(t) \phi_{i}(x)\right)^{2}
\end{aligned}
$$




$$
\begin{aligned}
& * \alpha_{3} \Gamma_{1} \sum_{i=1}^{N} p_{i}(t) \phi_{i}^{\prime \prime}(x) \\
& +\left(\frac{d_{2}}{d_{1}}+\sum_{i=1}^{N} p_{i}(t) \phi_{i}(x)-\sum_{i=1}^{N} q_{i}(t) \phi_{i}(x)\right)^{2} \\
& \cdot \alpha_{4}\left(V_{\mathrm{DC}}+V_{\mathrm{AC}} \cos (\Omega t)\right)^{2} \\
& -\left(1-\sum_{i=1}^{N} p_{i}(t) \phi_{i}(x)\right)^{2} \\
& \left.\cdot \alpha_{4}\left(V_{\mathrm{DC}}+V_{\mathrm{AC}} \cos (\Omega t)\right)^{2}\right) d x \\
& \int_{x=0}^{1} \phi_{j}(x)\left(\frac{d_{2}}{d_{1}}+\sum_{i=1}^{N} p_{i}(t) \phi_{i}(x)-\sum_{i=1}^{N} q_{i}(t) \phi_{i}(x)\right)^{2} \\
& *\left(\sum_{i=1}^{N} q_{i}(t) \phi_{i}^{i v}(x)+\sum_{i=1}^{N} \ddot{q}_{i}(t) \phi_{i}(x)\right. \\
& \left.+c \sum_{i=1}^{N} \dot{q}_{i}(t) \phi_{i}(x)\right) d x=\int_{x=0}^{1} \phi_{j}(x) \\
& \cdot\left(\left(\frac{d_{2}}{d_{1}}+\sum_{i=1}^{N} p_{i}(t) \phi_{i}(x)-\sum_{i=1}^{N} q_{i}(t) \phi_{i}(x)\right)^{2}\right. \\
& * \alpha_{3} \Gamma_{2} \sum_{i=1}^{N} q_{i}(t) \phi_{i}^{\prime \prime}(x) \\
& \left.+\alpha_{4}\left(V_{\mathrm{DC}}+V_{\mathrm{AC}} \cos (\Omega t)\right)^{2}\right) d x
\end{aligned}
$$

\section{Perturbation Analysis}

Numerical solutions of the ROM dynamical equations provide good results for low nonlinearity values. However, when tuning the system's geometrical parameters as well as the forcing amplitudes, one can increase the source of the nonlinearity, leading to nonlinear frequency responses which cannot be fully captured by ROM. As a result, perturbation theory was investigated to find the resonance of the double-microbeams especially for any high nonlinearity factors. The method of multiple scales was used by direct attack of the equations of motion $[6,24]$. So, the variable for the time scales and its derivatives were defined as follows:

$$
\begin{aligned}
& T_{0}=t, \\
& D_{0}=\frac{\partial}{\partial T_{0}}, \\
& T_{1}=\varepsilon t,
\end{aligned}
$$

$$
\begin{aligned}
& D_{1}=\frac{\partial}{\partial T_{1}}, \\
& T_{2}=\varepsilon^{2} t, \\
& D_{2}=\frac{\partial}{\partial T_{2}} .
\end{aligned}
$$

Next, the damping coefficient $c$ and the forcing amplitude $V_{\mathrm{AC}}$ were scaled so that their nonlinearity effect will be balanced in the modulation equations $[6,24]$ as follows:

$$
\begin{aligned}
c & =\varepsilon^{2} c, \\
V_{\mathrm{AC}} & =\varepsilon^{3} V_{\mathrm{AC}},
\end{aligned}
$$

where $\varepsilon$ is a bookkeeping parameter.

We seek a solution for the lower and upper electrodes in the following form, respectively:

$$
\begin{aligned}
w_{1}(x, t, \varepsilon)= & w_{s 1}(x)+u_{1}(x, t) \\
= & w_{s 1}(x)+\varepsilon u_{11}\left(x, T_{0}, T_{2}\right) \\
& +\varepsilon^{2} u_{12}\left(x, T_{0}, T_{2}\right)+\varepsilon^{3} u_{13}\left(x, T_{0}, T_{2}\right) \\
& +\cdots, \\
w_{2}(x, t, \varepsilon)= & w_{s 2}(x)+u_{2}(x, t) \\
= & w_{s 2}(x)+\varepsilon u_{21}\left(x, T_{0}, T_{2}\right) \\
& +\varepsilon^{2} u_{22}\left(x, T_{0}, T_{2}\right)+\varepsilon^{3} u_{23}\left(x, T_{0}, T_{2}\right) \\
& +\cdots,
\end{aligned}
$$

where $w_{s 1}$ and $w_{s 2}$ are the static components of the microbeams' deflection and $u_{1}$ and $u_{2}$ are their dynamic components for the lower and upper microbeams, respectively.

Substituting (14) and (15) into (5) and (6) gives

$$
\begin{aligned}
& \frac{\partial^{4} w_{s 1}}{\partial x^{4}}+ \frac{\partial^{4} u_{1}}{\partial x^{4}}+\frac{\partial^{2} u_{1}}{\partial t^{2}}+c \frac{\partial u_{1}}{\partial t} \\
&= \alpha_{3} \Gamma\left(w_{s 1}+u_{1}, w_{s 1}+u_{1}\right)\left(\frac{\partial^{2} w_{s 1}}{\partial x^{2}}+\frac{\partial^{2} u_{1}}{\partial x^{2}}\right) \\
&+\frac{\alpha_{4}\left(V_{\mathrm{DC}}+V_{\mathrm{AC}} \cos (\Omega t)\right)^{2}}{\left(1-w_{s 1}-u_{1}\right)^{2}} \\
&-\frac{\alpha_{4}\left(V_{\mathrm{DC}}+V_{\mathrm{AC}} \cos (\Omega t)\right)^{2}}{\left(d_{2} / d_{1}+w_{s 1}+u_{1}-w_{s 2}-u_{2}\right)^{2}}, \\
& \frac{\partial^{4} w_{s 2}}{\partial x^{4}}+\frac{\partial^{4} u_{2}}{\partial x^{4}}+\frac{\partial^{2} u_{2}}{\partial t^{2}}+c \frac{\partial u_{2}}{\partial t} \\
&=\alpha_{3} \Gamma\left(w_{s 2}+u_{2}, w_{s 2}+u_{2}\right)\left(\frac{\partial^{2} w_{s 2}}{\partial x^{2}}+\frac{\partial^{2} u_{2}}{\partial x^{2}}\right) \\
&+\frac{\alpha_{4}\left(V_{\mathrm{DC}}+V_{\mathrm{AC}} \cos (\Omega t)\right)^{2}}{\left(d_{2} / d_{1}+w_{s 1}+u_{1}-w_{s 2}-u_{2}\right)^{2}} .
\end{aligned}
$$


The electrical forces $\alpha_{4}\left(V_{\mathrm{DC}}+V_{\mathrm{AC}} \cos (\Omega t)\right)^{2} /\left(1-w_{s 1}-u_{1}\right)^{2}$ and $\alpha_{4}\left(V_{\mathrm{DC}}+V_{\mathrm{AC}} \cos (\Omega t)\right)^{2} /\left(d_{2} / d_{1}+w_{s 1}+u_{1}-w_{s 2}-\right.$ $\left.u_{2}\right)^{2}$ are expanded around $\left(u_{1}\right)$ and $\left(u_{1}-u_{2}\right)$, respectively, using a Taylor-series expansion. Further, the expression of $\left(V_{\mathrm{DC}}+V_{\mathrm{AC}} \cos (\Omega t)\right)^{2}$ will be approximated as $V_{\mathrm{DC}}^{2}+$ $2 V_{\mathrm{DC}} V_{\mathrm{AC}} \cos (\Omega t)$. Using the aforementioned expressions and canceling the static equations and neglecting all small terms while equating the coefficients of like powers of $\varepsilon$, the following equations are obtained:

For the upper microbeam, that is, (16),

(i) $\operatorname{order} \varepsilon^{1}$ :

$$
\mathscr{L}_{1}\left(u_{11}, u_{21}\right)=0 \text {, }
$$

(ii) $\operatorname{order} \varepsilon^{2}$ :

$$
\begin{aligned}
\mathscr{L}_{1}\left(u_{12}, u_{22}\right)= & \alpha_{3} w_{s 1}^{\prime \prime} \Gamma\left(u_{11}, u_{11}\right) \\
& +2 \alpha_{3} u_{11}^{\prime \prime} \Gamma\left(w_{s 1}, u_{11}\right)+\frac{3 \alpha_{4} V_{\mathrm{DC}}^{2} u_{11}^{2}}{\left(1-w_{s 1}\right)^{4}} \\
& -\frac{3 \alpha_{4} V_{\mathrm{DC}}^{2}\left(u_{11}^{2}-2 u_{11} u_{21}+u_{21}^{2}\right)}{\left(d_{2} / d_{1}+w_{s 1}-w_{s 2}\right)^{4}}
\end{aligned}
$$

(iii) $\operatorname{order} \varepsilon^{3}$ :

$$
\begin{aligned}
\mathscr{L}_{1}\left(u_{13}, u_{23}\right) & \\
= & -2 D_{0} D_{2} u_{11}-c D_{0} u_{11}+2 \alpha_{3} w_{s 1}^{\prime \prime} \Gamma\left(u_{11}, u_{12}\right) \\
& +2 \alpha_{3} u_{11}^{\prime \prime} \Gamma\left(w_{s 1}, u_{12}\right)+2 \alpha_{3} u_{12}^{\prime \prime} \Gamma\left(w_{s 1}, u_{11}\right) \\
& +\alpha_{3} \Gamma\left(u_{11}, u_{11}\right) u_{11}^{\prime \prime}+\frac{6 \alpha_{4} V_{\mathrm{DC}}^{2} u_{11} u_{12}}{\left(1-w_{s 1}\right)^{4}} \\
& +\frac{4 \alpha_{4} V_{\mathrm{DC}}^{2} u_{11}^{3}}{\left(1-w_{s 1}\right)^{5}} \\
+ & \frac{6 \alpha_{4} V_{\mathrm{DC}}^{2}\left(-u_{11} u_{12}+u_{11} u_{22}+u_{12} u_{21}-u_{21} u_{22}\right)}{\left(d_{2} / d_{1}+w_{s 1}-w_{s 2}\right)^{4}} \\
+ & \frac{4 \alpha_{4} V_{\mathrm{DC}}^{2}\left(u_{11}^{3}-3 u_{11}^{2} u_{21}+3 u_{11} u_{21}^{2}-u_{21}^{3}\right)}{\left(d_{2} / d_{1}+w_{s 1}-w_{s 2}\right)^{5}} \\
+ & 2 \widetilde{F}_{1} \cos \left(\Omega T_{0}\right)-2 \widetilde{F}_{2} \cos \left(\Omega T_{0}\right)
\end{aligned}
$$

where $\mathscr{L}_{1}$ is a linear differential operator defined for any two functions ( $f$ and $g$ ) by

$$
\begin{aligned}
\mathscr{L}_{1}(f, g)= & D_{0}^{2} f+f^{i v}-\alpha_{3} \Gamma\left(w_{s 1}, w_{s 1}\right) f^{\prime \prime} \\
& -2 \alpha_{3} w_{s 1}^{\prime \prime} \Gamma\left(w_{s 1}, f\right)-\frac{2 \alpha_{4} V_{\mathrm{DC}}^{2} f}{\left(1-w_{s 1}\right)^{3}}
\end{aligned}
$$

$$
\begin{aligned}
& -\frac{2 \alpha_{4} V_{\mathrm{DC}}^{2} f}{\left(d_{2} / d_{1}+w_{s 1}-w_{s 2}\right)^{3}} \\
& +\frac{2 \alpha_{4} V_{\mathrm{DC}}^{2} g}{\left(d_{2} / d_{1}+w_{s 1}-w_{s 2}\right)^{3}}=0,
\end{aligned}
$$

and where

$$
\begin{aligned}
& \widetilde{F}_{1}=\frac{\alpha_{4} V_{\mathrm{DC}} V_{\mathrm{AC}}}{\left(1-w_{s 1}\right)^{2}} \\
& \widetilde{F}_{2}=\frac{\alpha_{4} V_{\mathrm{DC}} V_{\mathrm{AC}}}{\left(\left(d_{2} / d_{1}\right)+w_{s 1}-w_{s 2}\right)^{2}} .
\end{aligned}
$$

For the upper microbeam, that is, (17),

(i) order $\varepsilon^{1}$ :

$$
\mathscr{L}_{2}\left(u_{11}, u_{21}\right)=0 \text {, }
$$

(ii) order $\varepsilon^{2}$ :

$$
\begin{aligned}
\mathscr{L}_{2}\left(u_{12}, u_{22}\right)= & \alpha_{3} w_{s 2}^{\prime \prime} \Gamma\left(u_{21}, u_{21}\right) \\
& +2 \alpha_{3} u_{21}^{\prime \prime} \Gamma\left(w_{s 2}, u_{21}\right) \\
& +\frac{3 \alpha_{4} V_{\mathrm{DC}}^{2}\left(u_{11}^{2}-2 u_{11} u_{21}+u_{21}^{2}\right)}{\left(d_{2} / d_{1}+w_{s 1}-w_{s 2}\right)^{4}},
\end{aligned}
$$

(iii) $\operatorname{order} \varepsilon^{3}$ :

$$
\begin{aligned}
\mathscr{L}_{2}\left(u_{13}, u_{23}\right) & \\
= & -2 D_{0} D_{2} u_{21}-c D_{0} u_{21}+2 \alpha_{3} w_{s 2}^{\prime \prime} \Gamma\left(u_{21}, u_{22}\right) \\
& +2 \alpha_{3} u_{21}^{\prime \prime} \Gamma\left(w_{s 2}, u_{22}\right)+2 \alpha_{3} u_{22}^{\prime \prime} \Gamma\left(w_{s 2}, u_{21}\right) \\
& +\alpha_{3} \Gamma\left(u_{21}, u_{21}\right) u_{21}^{\prime \prime} \\
& +\frac{6 \alpha_{4} V_{\mathrm{DC}}^{2}\left(u_{11} u_{12}-u_{11} u_{22}-u_{12} u_{21}+u_{21} u_{22}\right)}{\left(d_{2} / d_{1}+w_{s 1}-w_{s 2}\right)^{4}} \\
& +\frac{4 \alpha_{4} V_{\mathrm{DC}}^{2}\left(-u_{11}^{3}+3 u_{11}^{2} u_{21}-3 u_{11} u_{21}^{2}+u_{21}^{3}\right)}{\left(d_{2} / d_{1}+w_{s 1}-w_{s 2}\right)^{5}} \\
+ & 2 \widetilde{F}_{2} \cos \left(\Omega T_{0}\right),
\end{aligned}
$$

where $\mathscr{L}_{2}$ is a linear differential operator defined for any two functions $(f$ and $g$ ) by

$$
\begin{aligned}
\mathscr{L}_{2}(f, g)= & D_{0}{ }^{2} g+g^{i v}-\alpha_{3} \Gamma\left(w_{s 2}, w_{s 2}\right) g^{\prime \prime} \\
& -2 \alpha_{3} w_{s 2}^{\prime \prime} \Gamma\left(w_{s 2}, g\right) \\
& +\frac{2 \alpha_{4} V_{\mathrm{DC}}^{2} f}{\left(d_{2} / d_{1}+w_{s 1}-w_{s 2}\right)^{3}} \\
& -\frac{2 \alpha_{4} V_{\mathrm{DC}}^{2} g}{\left(d_{2} / d_{1}+w_{s 1}-w_{s 2}\right)^{3}} .
\end{aligned}
$$


Assuming no internal resonances situations, the solutions of (18) and (23) are assumed to consist of only the directly excited modes, $\Phi(x)$, since the indirectly excited modes will die out in the presence of damping. As a result, the solutions of the dynamic components $u_{11}$ and $u_{21}$ of the lower and upper microbeams, respectively, are

$$
\begin{aligned}
u_{11} & \left(x, T_{0}, T_{2}\right) \\
& =\left[A\left(T_{2}\right) e^{i \omega T_{0}}+\bar{A}\left(T_{2}\right) e^{-i \omega T_{0}}\right] \Phi_{1}(x), \\
u_{21} & \left(x, T_{0}, T_{2}\right) \\
& =\left[A\left(T_{2}\right) e^{i \omega T_{0}}+\bar{A}\left(T_{2}\right) e^{-i \omega T_{0}}\right] \Phi_{2}(x),
\end{aligned}
$$

where $A\left(T_{2}\right)$ is a complex-valued function that is determined by imposing the solvability condition at the third order, the overbar denotes the complex conjugate, and $\omega$ and $\Phi(x)$ are the natural frequency and corresponding eigenfunction of the directly excited modes, respectively. Here, the complexvalued function is considered to be the same for both microbeams, while the mode shapes will compensate for the resulting difference. This will help in obtaining the eigenvalue problem. Substituting (27) and (28) into (18) and (23) we obtain the following nonlinear coupled eigenvalue problem (EVP):

$$
\begin{aligned}
& -\omega^{2} \Phi_{1}+\Phi_{1}^{i v}-\alpha_{3} \Gamma\left(w_{s 1}, w_{s 1}\right) \Phi_{1}^{\prime \prime} \\
& -2 \alpha_{3} w_{s 1}^{\prime \prime} \Gamma\left(w_{s 1}, \Phi_{1}\right)-\frac{2 \alpha_{4} V_{\mathrm{DC}}^{2} \Phi_{1}}{\left(1-w_{s 1}\right)^{3}} \\
& -\frac{2 \alpha_{4} V_{\mathrm{DC}}^{2} \Phi_{1}}{\left(d_{2} / d_{1}+w_{s 1}-w_{s 2}\right)^{3}} \\
& +\frac{2 \alpha_{4} V_{\mathrm{DC}}^{2} \Phi_{2}}{\left(d_{2} / d_{1}+w_{s 1}-w_{s 2}\right)^{3}}=0, \\
& -\omega^{2} \Phi_{2}+\Phi_{2}^{i v}-\alpha_{3} \Gamma\left(w_{s 2}, w_{s 2}\right) \Phi_{2}^{\prime \prime} \\
& -2 \alpha_{3} w_{s 2}^{\prime \prime} \Gamma\left(w_{s 2}, \Phi_{2}\right)-\frac{2 \alpha_{4} V_{\mathrm{DC}}^{2} \Phi_{2}}{\left(d_{2} / d_{1}+w_{s 1}-w_{s 2}\right)^{3}} \\
& +\frac{2 \alpha_{4} V_{\mathrm{DC}}^{2} \Phi_{1}}{\left(d_{2} / d_{1}+w_{s 1}-w_{s 2}\right)^{3}}=0 .
\end{aligned}
$$

Next, the solutions $u_{11}$ and $u_{21}$ will be substituted in the second-order equations $\left(\varepsilon^{2}\right)$, which will give

$$
\begin{aligned}
& \mathscr{L}_{1}\left(u_{12}, u_{22}\right) \\
& =\left(A^{2} e^{2 i \omega T_{0}}+2 A \bar{A}+\bar{A}^{2} e^{-2 i \omega T_{0}}\right) h_{1}(x), \\
& \mathscr{L}_{2}\left(u_{12}, u_{22}\right) \\
& =\left(A^{2} e^{2 i \omega T_{0}}+2 A \bar{A}+\bar{A}^{2} e^{-2 i \omega T_{0}}\right) h_{2}(x),
\end{aligned}
$$

where

$$
\begin{aligned}
h_{1}(x)= & \alpha_{3} w_{s 1}^{\prime \prime} \Gamma\left(\Phi_{1}, \Phi_{1}\right)+2 \alpha_{3} \Phi_{1}^{\prime \prime} \Gamma\left(w_{s 1}, \Phi_{1}\right) \\
& +\frac{3 \alpha_{4} V_{\mathrm{DC}}^{2} \Phi_{1}^{2}}{\left(1-w_{s 1}\right)^{4}}-\frac{3 \alpha_{4} V_{\mathrm{DC}}^{2} \Phi_{1}{ }^{2}}{\left(d_{2} / d_{1}+w_{s 1}-w_{s 2}\right)^{4}} \\
& +\frac{6 \alpha_{4} V_{\mathrm{DC}}^{2} \Phi_{1} \Phi_{2}}{\left(d_{2} / d_{1}+w_{s 1}-w_{s 2}\right)^{4}} \\
& -\frac{3 \alpha_{4} V_{\mathrm{DC}}^{2} \Phi_{2}^{2}}{\left(d_{2} / d_{1}+w_{s 1}-w_{s 2}\right)^{4}}, \\
h_{2}(x)= & \alpha_{3} w_{s 2}^{\prime \prime} \Gamma\left(\Phi_{2}, \Phi_{2}\right)+2 \alpha_{3} \Phi_{2}^{\prime \prime} \Gamma\left(w_{s 2}, \Phi_{2}\right) \\
& +\frac{3 \alpha_{4} V_{\mathrm{DC}}^{2} \Phi_{1}^{2}}{\left(d_{2} / d_{1}+w_{s 1}-w_{s 2}\right)^{4}} \\
& -\frac{6 \alpha_{4} V_{\mathrm{DC}}^{2} \Phi_{1} \Phi_{2}}{\left(d_{2} / d_{1}+w_{s 1}-w_{s 2}\right)^{4}} \\
& +\frac{3 \alpha_{4} V_{\mathrm{DC}}^{2} \Phi_{2}{ }^{2}}{\left(d_{2} / d_{1}+w_{s 1}-w_{s 2}\right)^{4}} .
\end{aligned}
$$

Assume the particular solution of $u_{12}$ and $u_{22}$ as

$$
\begin{aligned}
& u_{12}=\left(A^{2} e^{2 i \omega T_{0}} \Psi_{11}+2 A \bar{A} \Psi_{12}+\bar{A}^{2} e^{-2 i \omega T_{0}} \Psi_{11}\right), \\
& u_{22}=\left(A^{2} e^{2 i \omega T_{0}} \Psi_{21}+2 A \bar{A} \Psi_{22}+\bar{A}^{2} e^{-2 i \omega T_{0}} \Psi_{21}\right),
\end{aligned}
$$

where the functions $\Psi_{i j}$ are the solutions of the boundary value problems:

$$
\begin{aligned}
M_{1}\left(\Psi_{1 i}, 2 \omega \delta_{1 i}\right) & =h_{1}(x), \\
M_{2}\left(\Psi_{2 i}, 2 \omega \delta_{1 i}\right) & =h_{2}(x), \\
\Psi_{1 j} & =0 ; \\
\Psi_{1 j}^{\prime} & =0 ; \\
\Psi_{2 j} & =0 ; \\
\Psi_{2 j}^{\prime} & =0 ;
\end{aligned}
$$

all evaluated at $x=0, x=1$ for $j=1,2$,

and where $\delta$ is the Kronecker delta operator and the two linear differential operators $M_{1}$ and $M_{2}$ are defined as

$$
\begin{aligned}
M_{1}\left(\Psi_{1 i}, \omega\right)= & \Psi_{1 i}^{i v}-\omega^{2} \Psi_{1 i}-\alpha_{3} \Gamma\left(w_{s 1}, w_{s 1}\right) \Psi_{1 i}^{\prime \prime} \\
& -2 \alpha_{3} w_{s 1}^{\prime \prime} \Gamma\left(w_{s 1}, \Psi_{1 i}\right)-\frac{2 \alpha_{4} V_{\mathrm{DC}}^{2} \Psi_{1 i}^{\prime}}{\left(1-w_{s 1}\right)^{3}}
\end{aligned}
$$




$$
\begin{aligned}
& -\frac{2 \alpha_{4} V_{\mathrm{DC}}^{2} \Psi_{1 i}}{\left(d_{2} / d_{1}+w_{s 1}-w_{s 2}\right)^{3}} \\
& +\frac{2 \alpha_{4} V_{\mathrm{DC}}^{2} \Psi_{2 i}}{\left(d_{2} / d_{1}+w_{s 1}-w_{s 2}\right)^{3}}, \\
M_{2}\left(\Psi_{2 i}, \omega\right)= & \Psi_{2 i}^{i v}-\omega^{2} \Psi_{2 i}-\alpha_{3} \Gamma\left(w_{s 2}, w_{s 2}\right) \Psi_{2 i}^{\prime \prime} \\
& -2 \alpha_{3} w_{s 2}^{\prime \prime} \Gamma\left(w_{s 2}, \Psi_{2 i}\right) \\
& +\frac{2 \alpha_{4} V_{\mathrm{DC}}^{2} \Psi_{1 i}}{\left(d_{2} / d_{1}+w_{s 1}-w_{s 2}\right)^{3}} \\
& -\frac{2 \alpha_{4} V_{\mathrm{DC}}^{2} \Psi_{2 i}}{\left(d_{2} / d_{1}+w_{s 1}-w_{s 2}\right)^{3}} .
\end{aligned}
$$

Introduce the detuning parameter $\sigma$ to describe the nearness of the excitation frequency $\Omega$ to the fundamental natural frequency of the excited mode $\omega$ as

$$
\Omega=\omega+\varepsilon^{2} \sigma .
$$

The solutions of the first and second order are then substituted in (20) and (25), leading to

$$
\begin{aligned}
& \mathscr{L}_{1}\left(u_{13}, u_{23}\right)=D_{0}{ }^{2} u_{13}+u_{13}^{i v}-\alpha_{3} \Gamma\left(w_{s 1}, w_{s 1}\right) u_{13}^{\prime \prime} \\
& -2 \alpha_{3} w_{s 1}^{\prime \prime} \Gamma\left(w_{s 1}, u_{13}\right)-\frac{2 \alpha_{4} V_{\mathrm{DC}}^{2} u_{13}}{\left(1-w_{s 1}\right)^{3}} \\
& -\frac{2 \alpha_{4} V_{\mathrm{DC}}^{2} u_{13}}{\left(d_{2} / d_{1}+w_{s 1}-w_{s 2}\right)^{3}}+\frac{2 \alpha_{4} V_{\mathrm{DC}}^{2} u_{23}}{\left(d_{2} / d_{1}+w_{s 1}-w_{s 2}\right)^{3}} \\
& =\left(-2 i \omega A^{\prime} \Phi_{1}(x)-i c \omega \Phi_{1}(x) A+\chi_{1}(x) A^{2} \bar{A}\right. \\
& \left.+\widetilde{F}_{1} e^{i \sigma_{1} T_{2}}-\widetilde{F}_{2} e^{i \sigma_{2} T_{2}}\right) e^{i \omega T_{0}}+c c+\mathrm{NST} ; \\
& \mathscr{L}_{2}\left(u_{13}, u_{23}\right)=D_{0}{ }^{2} u_{23}+u_{23}^{i v}-\alpha_{3} \Gamma\left(w_{s 2}, w_{s 2}\right) u_{23}^{\prime \prime} \\
& -2 \alpha_{3} w_{s 2}^{\prime \prime} \Gamma\left(w_{s 2}, u_{23}\right)+\frac{2 \alpha_{4} V_{\mathrm{DC}}^{2} u_{13}}{\left(d_{2} / d_{1}+w_{s 1}-w_{s 2}\right)^{3}} \\
& -\frac{2 \alpha_{4} V_{\mathrm{DC}}^{2} u_{23}}{\left(d_{2} / d_{1}+w_{s 1}-w_{s 2}\right)^{3}}=\left(-2 i \omega A^{\prime} \Phi_{2}(x)\right. \\
& \left.-i c \omega A \Phi_{2}(x)+A^{2} \bar{A} \chi_{2}(x)+\widetilde{F}_{2} e^{i \sigma_{2} T_{2}}\right) e^{i \omega T_{0}}+c c \\
& +\mathrm{NST},
\end{aligned}
$$

where NST stands for the nonsecular terms and the functions $\chi_{i}$ are defined as

$$
\begin{aligned}
& \chi_{1}(x)=2 \alpha_{3} w_{s 1}^{\prime \prime}\left(\Gamma\left(\Phi_{1}, \Psi_{11}\right)+2 \Gamma\left(\Phi_{1}, \Psi_{12}\right)\right) \\
& \quad+2 \alpha_{3} \Phi_{1}^{\prime \prime}\left(\Gamma\left(w_{s 1}, \Psi_{11}\right)+2 \Gamma\left(w_{s 1}, \Psi_{12}\right)\right) \\
& \quad+2 \alpha_{3} \Gamma\left(w_{s 1}, \Phi_{1}\right)\left(\Psi_{11}^{\prime \prime}+2 \Psi_{12}^{\prime \prime}\right)+3 \alpha_{3} \Gamma\left(\Phi_{1}, \Phi_{1}\right) \Phi_{1}^{\prime \prime}
\end{aligned}
$$

$$
\begin{aligned}
& +\frac{6 \alpha_{4} V_{\mathrm{DC}}^{2}}{\left(1-w_{s 1}\right)^{4}}\left(\Psi_{11} \Phi_{1}+2 \Psi_{12} \Phi_{1}\right)+\frac{12 \alpha_{4} V_{\mathrm{DC}}^{2}}{\left(1-w_{s 1}\right)^{5}} \Phi_{1}{ }^{3} \\
& +\frac{6 \alpha_{4} V_{\mathrm{DC}}^{2}}{\left(\left(d_{2} / d_{1}\right)+w_{s 1}-w_{s 2}\right)^{4}}\left(-\Phi_{1} \Psi_{11}-2 \Phi_{1} \Psi_{12}\right. \\
& +\Phi_{1} \Psi_{21}+2 \Phi_{1} \Psi_{22}+\Phi_{2} \Psi_{11}+2 \Phi_{2} \Psi_{12}-\Phi_{2} \Psi_{21} \\
& \left.-2 \Phi_{2} \Psi_{22}\right)+\frac{12 \alpha_{4} V_{\mathrm{DC}}^{2}}{\left(\left(d_{2} / d_{1}\right)+w_{s 1}-w_{s 2}\right)^{5}}\left(\Phi_{1}{ }^{3}\right. \\
& \left.-3 \Phi_{1}{ }^{2} \Phi_{2}+3 \Phi_{1} \Phi_{2}{ }^{2}-\Phi_{2}{ }^{3}\right) ; \\
& \chi_{2}(x)=2 \alpha_{3} w_{s 2}^{\prime \prime}\left(\Gamma\left(\Phi_{2}, \Psi_{21}\right)+2 \Gamma\left(\Phi_{2}, \Psi_{22}\right)\right) \\
& +2 \alpha_{3} \Phi_{2}^{\prime \prime}\left(\Gamma\left(w_{s 2}, \Psi_{21}\right)+2 \Gamma\left(w_{s 2}, \Psi_{22}\right)\right) \\
& +2 \alpha_{3} \Gamma\left(w_{s 2}, \Phi_{2}\right)\left(\Psi_{21}^{\prime \prime}+2 \Psi_{22}^{\prime \prime}\right)+3 \alpha_{3} \Gamma\left(\Phi_{2}, \Phi_{2}\right) \Phi_{2}^{\prime \prime} \\
& +\frac{6 \alpha_{4} V_{\mathrm{DC}}^{2}}{\left(\left(d_{2} / d_{1}\right)+w_{s 1}-w_{s 2}\right)^{4}}\left(\Phi_{1} \Psi_{11}+2 \Phi_{1} \Psi_{12}\right. \\
& -\Phi_{1} \Psi_{21}-2 \Phi_{1} \Psi_{22}-\Phi_{2} \Psi_{11}-2 \Phi_{2} \Psi_{12}+\Phi_{2} \Psi_{21} \\
& \left.+2 \Phi_{2} \Psi_{22}\right)+\frac{12 \alpha_{4} V_{\mathrm{DC}}^{2}}{\left(\left(d_{2} / d_{1}\right)+w_{s 1}-w_{s 2}\right)^{5}}\left(-\Phi_{1}{ }^{3}\right. \\
& \left.+3 \Phi_{1}{ }^{2} \Phi_{2}-3 \Phi_{1} \Phi_{2}{ }^{2}+\Phi_{2}{ }^{3}\right) .
\end{aligned}
$$

Hence, we need to eliminate the secular terms in (36) by seeking a particular solution free of secular terms in the form:

$$
\begin{aligned}
& u_{13}\left(x, T_{0}, T_{2}\right)=\Upsilon_{1}\left(x, T_{2}\right) e^{i \omega T_{0}} \\
& u_{23}\left(x, T_{0}, T_{2}\right)=\Upsilon_{2}\left(x, T_{2}\right) e^{i \omega T_{0}} .
\end{aligned}
$$

Then, (38) are substituted into (36). Next, the coefficients of $e^{i \omega T_{0}}$ in the resultant equations will be equated. The outcome of two equations will be then multiplied by two adjoints functions $P(x)$ and $Q(x)$, respectively, and then integrated by parts to transfer the derivatives from $\Upsilon$ to the adjoints, leading to

$$
\begin{aligned}
& \int_{x=0}^{1} \Upsilon_{1}\left(-\omega^{2} P+P^{i v}-\alpha_{3} \Gamma\left(w_{s 1}, w_{s 1}\right) P^{\prime \prime}\right. \\
& -2 \alpha_{3} w_{s 1}^{\prime \prime} \Gamma\left(P, w_{s 1}\right)-\frac{2 \alpha_{4} V_{\mathrm{DC}}^{2} P}{\left(1-w_{s 1}\right)^{3}} \\
& \left.-\frac{2 \alpha_{4} V_{\mathrm{DC}}^{2} P}{\left(d_{2} / d_{1}+w_{s 1}-w_{s 2}\right)^{3}}\right) d x+\left[P \Upsilon_{1}^{\prime \prime \prime}\right. \\
& -P^{\prime} \Upsilon_{1}^{\prime \prime}+P^{\prime \prime} \Upsilon_{1}^{\prime}-P^{\prime \prime \prime} \Upsilon_{1}-\alpha_{3} \Gamma\left(w_{s 1}, w_{s 1}\right)\left(P \Upsilon_{1}^{\prime}\right. \\
& \left.-P^{\prime} \Upsilon_{1}\right)+2 \alpha_{3} w_{s 1}^{\prime} P \int_{x=0}^{1} w_{s 1}^{\prime \prime} \Upsilon_{1} d x-2 \alpha_{3} w_{s 1}^{\prime 2} P \Upsilon_{1}
\end{aligned}
$$




$$
\begin{aligned}
& \left.+2 \alpha_{3} w_{s 1}^{\prime} \Upsilon_{1} \Gamma\left(P, w_{s 1}\right)\right]_{x=0}^{1}+\int_{x=0}^{1} \Upsilon_{2} \\
& \frac{2 \alpha_{4} V_{\mathrm{DC}}^{2} P}{\left(d_{2} / d_{1}+w_{s 1}-w_{s 2}\right)^{3}} d x \\
& =\int_{x=0}^{1} P\left(-2 i \omega A^{\prime} \Phi_{1}(x)-i c \omega \Phi_{1}(x) A\right. \\
& \left.+\chi_{1}(x) A^{2} \bar{A}+\widetilde{F}_{1} e^{i \sigma_{1} T_{2}}-\widetilde{F}_{2} e^{i \sigma_{2} T_{2}}\right) d x \\
& \int_{x=0}^{1} \Upsilon_{2}\left(-\omega^{2} Q+Q^{i v}-\alpha_{3} \Gamma\left(w_{s 2}, w_{s 2}\right) Q^{\prime \prime}\right. \\
& -2 \alpha_{3} w_{s 2}^{\prime \prime} \Gamma\left(Q, w_{s 2}\right) \\
& \left.-\frac{2 \alpha_{4} V_{\mathrm{DC}}^{2} Q}{\left(d_{2} / d_{1}+w_{s 1}-w_{s 2}\right)^{3}}\right) d x+\int_{x=0}^{1} \Upsilon_{1} \\
& \cdot \frac{2 \alpha_{4} V_{\mathrm{DC}}^{2} Q}{\left(d_{2} / d_{1}+w_{s 1}-w_{s 2}\right)^{3}} d x+\left[Q Y_{2}^{\prime \prime \prime}-Q^{\prime} Y_{2}^{\prime \prime}\right. \\
& +Q^{\prime \prime} \Upsilon_{2}^{\prime}-Q^{\prime \prime \prime} \Upsilon_{2}-\alpha_{3} \Gamma\left(w_{s 2}, w_{s 2}\right)\left(Q \Upsilon_{2}^{\prime}-Q^{\prime} \Upsilon_{2}\right) \\
& +2 \alpha_{3} w_{s 2}^{\prime} Q \int_{x=0}^{1} w_{s 2}^{\prime \prime} \Upsilon_{2} d x-2 \alpha_{3} w_{s 2}^{\prime 2} Q \Upsilon_{2} \\
& \left.+2 \alpha_{3} w_{s 2}^{\prime} \Upsilon_{2} \Gamma\left(Q, w_{s 2}\right)\right]_{x=0}^{1} \\
& =\int_{x=0}^{1} Q\left(-2 i \omega A^{\prime} \Phi_{2}(x)-i c \omega A \Phi_{2}(x)\right. \\
& \left.+A^{2} \bar{A} \chi_{2}(x)+\widetilde{F}_{2} e^{i \sigma_{2} T_{2}}\right) d x \text {. }
\end{aligned}
$$

By adding (39) and rearranging the terms, we can obtain the two adjoints equations governing both functions $P(x)$ and $Q(x)$ as follows:

$$
\begin{aligned}
- & \omega^{2} P+P^{i v}-\alpha_{3} \Gamma\left(w_{s 1}, w_{s 1}\right) P^{\prime \prime}-2 \alpha_{3} w_{s 1}^{\prime \prime} \Gamma\left(P, w_{s 1}\right) \\
- & \frac{2 \alpha_{4} V_{\mathrm{DC}}^{2} P}{\left(1-w_{s 1}\right)^{3}}-\frac{2 \alpha_{4} V_{\mathrm{DC}}^{2} P}{\left(d_{2} / d_{1}+w_{s 1}-w_{s 2}\right)^{3}} \\
& +\frac{2 \alpha_{4} V_{\mathrm{DC}}^{2} Q}{\left(d_{2} / d_{1}+w_{s 1}-w_{s 2}\right)^{3}}=0 ; \\
- & \omega^{2} \mathrm{Q}+Q^{i v}-\alpha_{3} \Gamma\left(w_{s 2}, w_{s 2}\right) Q^{\prime \prime}-2 \alpha_{3} w_{s 2}^{\prime \prime} \Gamma\left(Q, w_{s 2}\right) \\
- & \frac{2 \alpha_{4} V_{\mathrm{DC}}^{2} Q}{\left(d_{2} / d_{1}+w_{s 1}-w_{s 2}\right)^{3}} \\
+ & \frac{2 \alpha_{4} V_{\mathrm{DC}}^{2} P}{\left(d_{2} / d_{1}+w_{s 1}-w_{s 2}\right)^{3}}=0
\end{aligned}
$$

which are the same as the first-order equations (29) so we call them self-adjoint equations. The solvability condition can be then obtained as

$$
\begin{aligned}
& \int_{x=0}^{1}\left(-2 i \omega A^{\prime}\left(P \Phi_{1}+Q \Phi_{2}\right)-i c \omega A\left(P \Phi_{1}+Q \Phi_{2}\right)\right. \\
& +A^{2} \bar{A}\left(P \chi_{1}+Q \chi_{2}\right)+\widetilde{F}_{1} P e^{i \sigma_{1} T_{2}} \\
& \left.\quad+\widetilde{F}_{2} e^{i \sigma_{2} T_{2}}(Q-P)\right) d x=0 .
\end{aligned}
$$

Using Euler's formula for $e^{i \sigma_{1} T_{2}}$ and $e^{i \sigma_{2} T_{2}}$, then separating the real and imaginary parts of the solvability condition will give the following two coupled equations:

$$
\begin{aligned}
& \beta^{\prime} a\left(z_{1}+z_{2}\right)+\frac{1}{8 \omega} a^{3}\left(S_{1}+S_{2}\right) \\
& =-\frac{F_{1}}{\omega} \cos \gamma_{1}-\frac{1}{\omega}\left(F_{22}-F_{21}\right) \cos \gamma_{2}, \\
& a^{\prime}\left(z_{1}+z_{2}\right)+\frac{c}{2} a\left(z_{1}+z_{2}\right) \\
& =\frac{F_{1}}{\omega} \sin \gamma_{1}+\frac{1}{\omega}\left(F_{22}-F_{21}\right) \sin \gamma_{2},
\end{aligned}
$$

where

$$
\begin{aligned}
z_{1} & =\int_{x=0}^{1} P \Phi_{1} d x, \\
z_{2} & =\int_{x=0}^{1} Q \Phi_{2} d x, \\
S_{1} & =\int_{x=0}^{1} P \chi_{1} d x, \\
S_{2} & =\int_{x=0}^{1} Q \chi_{2} d x, \\
F_{1} & =\int_{x=0}^{1} \widetilde{F}_{1} P d x, \\
F_{21} & =\int_{x=0}^{1} \widetilde{F}_{2} P d x, \\
F_{22} & =\int_{x=0}^{1} \widetilde{F}_{2} Q d x, \\
\gamma & =\sigma T_{2}-\beta .
\end{aligned}
$$

By squaring both sides of (42) and (43) and then adding the results, while remembering that $\left(\beta^{\prime}=\sigma-\gamma^{\prime}\right)$, we get

$$
\begin{aligned}
& a_{0}^{2}\left(\left(\sigma\left(z_{1}+z_{2}\right)+\frac{1}{8 \omega} a_{0}^{2}\left(S_{1}+S_{2}\right)\right)^{2}\right. \\
& \left.\quad+\frac{c^{2}}{4}\left(z_{1}+z_{2}\right)^{2}\right)=\frac{\left(F_{1}+F_{22}-F_{21}\right)^{2}}{\omega^{2}} .
\end{aligned}
$$

Since the quality factor is related to the damping coefficient (c) by

$$
Q=\frac{\omega}{c\left(z_{1}+z_{2}\right)}
$$




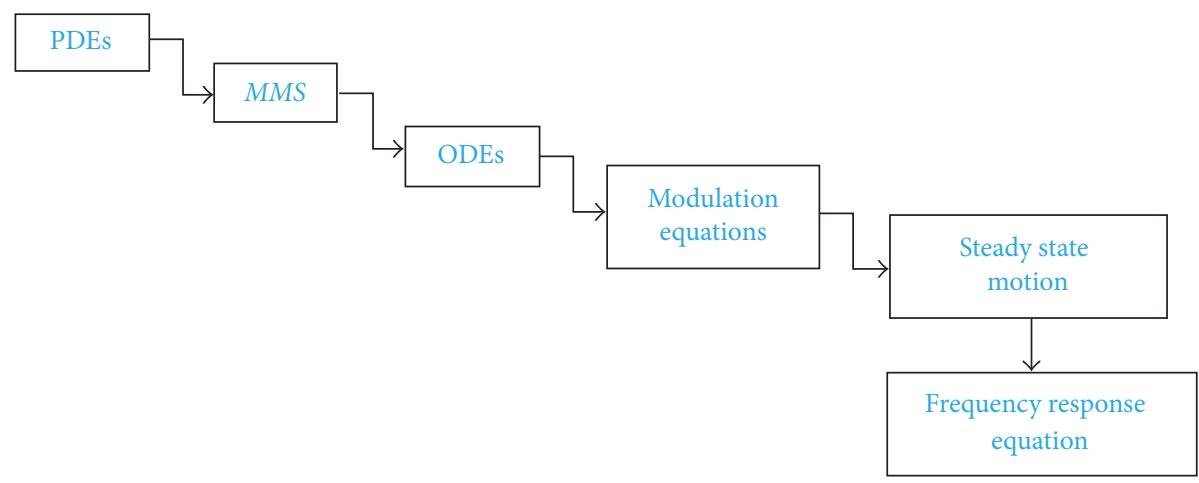

Figure 2

it can be replaced in the solvability equation (45) to examine the effect of the quality factor on the system dynamics.

\section{ROM and Perturbation Results}

To solve for the coupled equations of motion of both the lower and upper microbeams using the ROM, it is necessary to solve for the unknown functions $p_{i}(t)$ and $q_{i}(t)$ in (11) and then substituting them back into (9) and (10), which will give the deflection of both microbeams.

For the method of multiple scales based perturbation technique, we start first by scaling the first-order coupled equations, (29) for both functions $\Phi_{1}$ and $\Phi_{2}$. Then, we substitute them into the boundary value problems equations (33) to solve for the unknown functions $\Psi_{11}, \Psi_{12}, \Psi_{21}$, and $\Psi_{22}$. Then, we solve for the unknown dynamic amplitude from (45) and the phase from either (42) or (43). Finally, the deflection of the lower and upper microbeams can be calculated using (14) and (15), respectively. More details about this numerical resolution technique can be found in [16]. Figure 2 is also accommodating the above numerical method description in summarizing the sequence of operations done in this particular method of multiple scales (MMS) technique.

Since each two consecutive natural frequencies of this system are very close to each other, the equations of the perturbation will be solved twice for each one of those two, and then the deflections around the two natural frequencies for each microbeam will be added to give the resultant deflections of both microbeams. A comparison of the obtained results for the two above described methods for the case of doublemicrobeams is shown in Figures 3-5. The selected parameters are the same as assumed in the static analysis [18] and shown in Table 1, with the only difference being the applied voltages, $V_{\mathrm{DC}}=10$ Volts and $V_{\mathrm{AC}}=0.5$ Volts. In this present case, it can be seen from all figures that overall both assumed numerical methods are in good agreement for all different cases. While the perturbation analysis technique is less accurate than the ROM, it showed better global results in the presence of gross nonlinearity. It can predict all of the stable and unstable branches which the ROM is not capable of capturing. Also, it gives a better global and local dynamical picture about the microsystem's behavior. Hence, the perturbation technique will be used in the following parametric study.

\section{Parametric Study}

In order to investigate the effect of different physical and geometrical parameters on the dynamical amplitude of the double-microbeams based actuator, three different values were selected (low, medium, and high) for each chosen parameter; then the frequency response curve was generated for each microbeam to investigate the effects of each tuned parameter. The selected parameters are the length, the quality factor, and the DC and AC voltages. For all those cases, the depths of both air gaps are kept equal $\left(d_{1}=d_{2}=1.25 \mu \mathrm{m}\right)$ and the width $(b)$ and height $(h)$ for both microbeams are initially assumed to be $20 \mu \mathrm{m}$ and $1.5 \mu \mathrm{m}$, respectively. The length $(L)$ and the quality factor $(Q)$ were equal to $210 \mu \mathrm{m}$ and 50, respectively, for all the cases except when they are intentionally varied.

6.1. Effect of the Actuator Length (L). Figure 6 shows the amplitude versus the excitation frequency for three different lengths $(210 \mu \mathrm{m}, 410 \mu \mathrm{m}$, and $610 \mu \mathrm{m})$. From both figures, the significance of changing the length on the dynamical amplitude, even when small voltages were applied (i.e., $V_{\mathrm{DC}}=$ 2 Volts and $V_{\mathrm{AC}}=0.1$ Volts), is clear. When the length is of a small value $(L=210 \mu \mathrm{m})$, the two resonance peaks are very close to each other, which means that the applied voltages have a slight effect on the microstructure and so do not change its fundamental frequencies. However, when the value of the microbeam length is increased ( $L=410$ and $610 \mu \mathrm{m})$, the distance between the two peaks becomes larger, meaning the fundamental frequency becomes lowered and the system is prone to experience a pull-in instability. Also, with a high value of the microbeam length, the nonlinearity becomes more dominant, producing a softening-type of behavior at the fundamental frequency for both microbeams.

6.2. Effect of the Quality Factor (Q). Figure 7 shows the amplitude versus the excitation frequency for three different quality factors $(50,150$, and 500) at high DC and AC voltages (i.e., $V_{\mathrm{DC}}=10$ Volts and $V_{\mathrm{AC}}=0.5$ Volts). It can be noticed that there is no effect for the quality factor on the resonance locations, which is reasonable since increasing the quality factor will decrease the damping which has no effect on the 


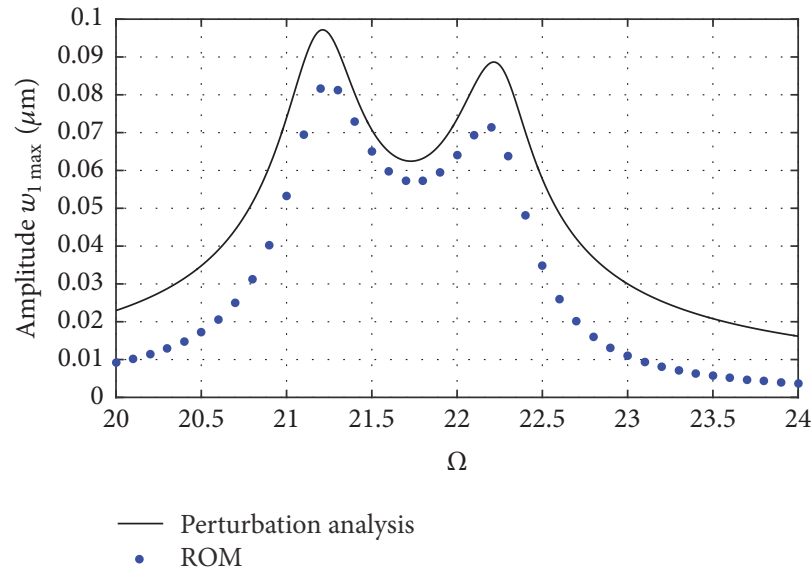

(a)

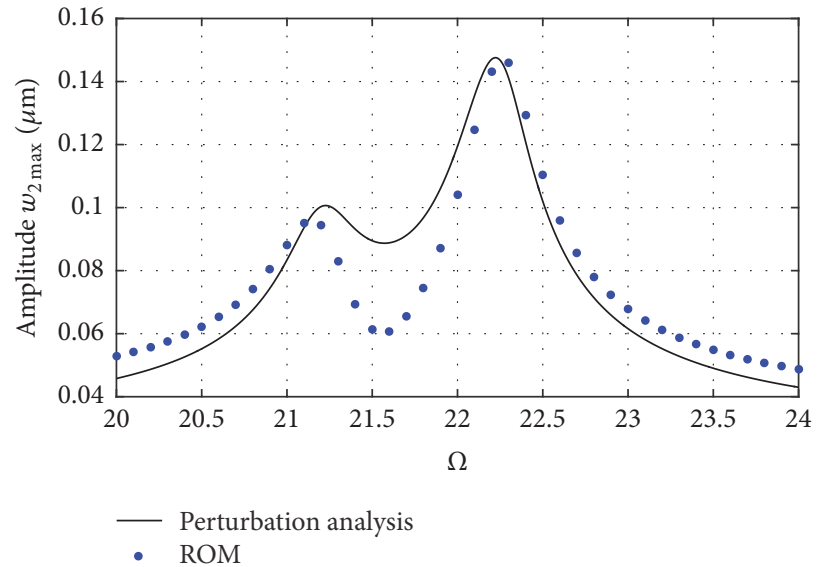

(b)

FIGURE 3: Frequency response curves of the (a) upper microbeam and (b) lower microbeam assuming both ROM and perturbation method for the case of $d_{1}=d_{2}$.

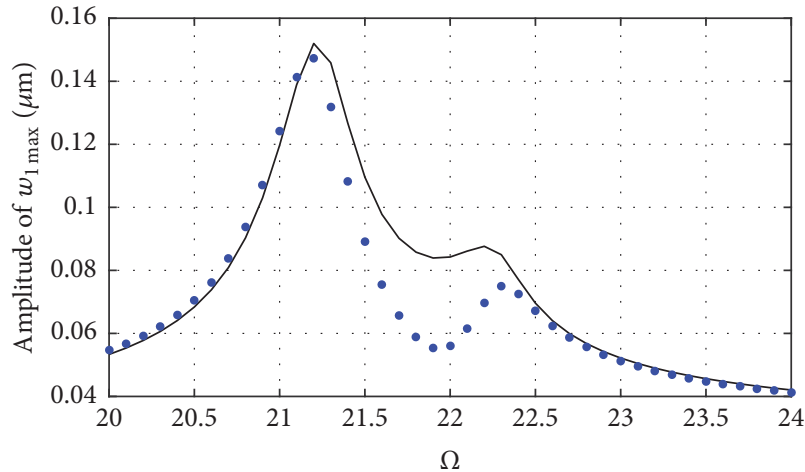

- Perturbation analysis

- $\mathrm{ROM}$

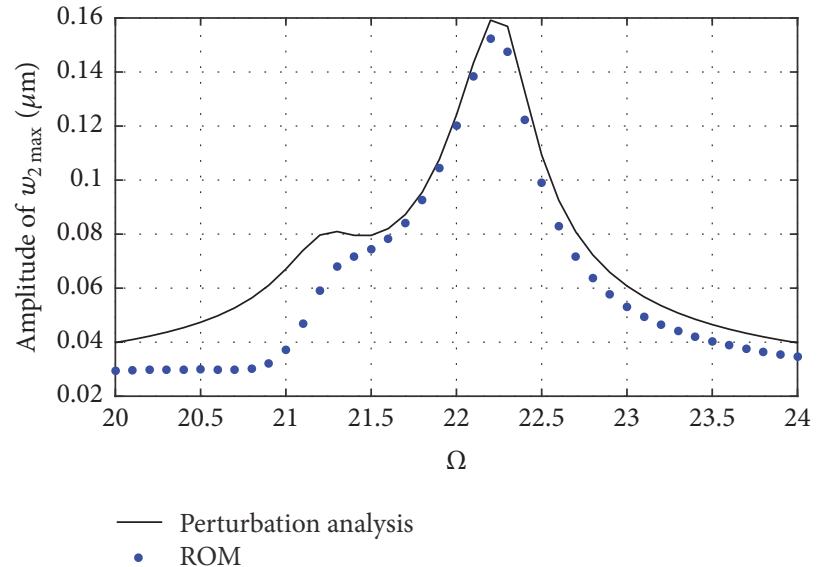

(b)

FIGURE 4: Frequency response curves of the (a) upper microbeam and (b) lower microbeam assuming both ROM and perturbation method for the case of $d_{1}<d_{2}$.

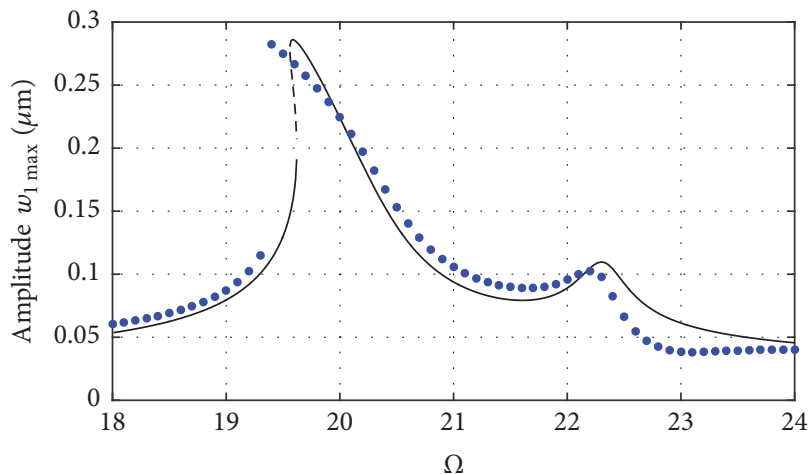

_ Perturbation analysis - ROM

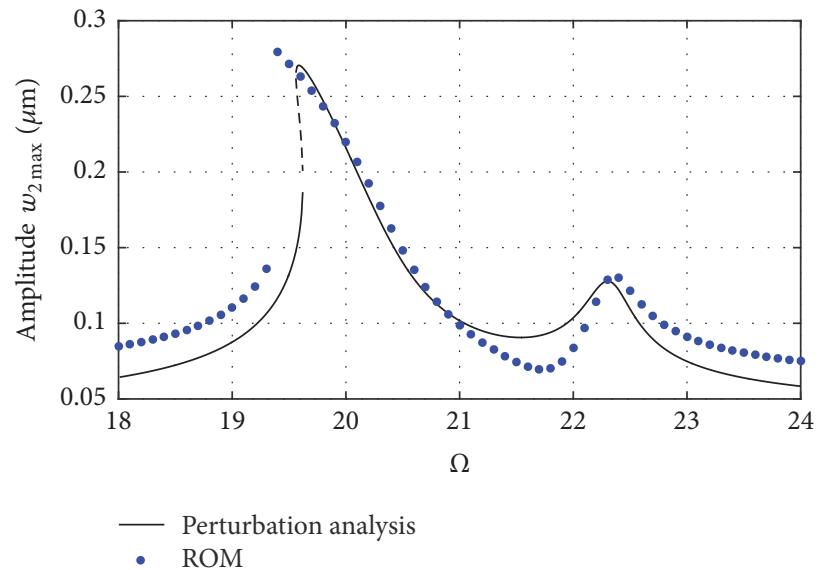

(b)

FIGURE 5: Frequency response curves of the (a) upper microbeam and (b) lower microbeam assuming both ROM and perturbation method for the case of $d_{1}>d_{2}$. 
TABLE 1: Assumed geometrical and material properties.

\begin{tabular}{|c|c|c|c|c|c|}
\hline Parameter & Value & Parameter & \multicolumn{3}{|c|}{ Value } \\
\hline Beam length $(L)$ & $150 \mu \mathrm{m}$ & Effective Young's modulus $(E)$ & \multicolumn{3}{|c|}{$166 \mathrm{GPa}$} \\
\hline Beam thickness $(h)$ & $1.0 \mu \mathrm{m}$ & Density $(\rho)$ & \multicolumn{3}{|c|}{$2,332 \mathrm{~kg} / \mathrm{m}^{3}$} \\
\hline Beam width $(b)$ & $4.0 \mu \mathrm{m}$ & Air gap depths $\left(d_{1}\right.$ and $d_{2}$, resp.) & $\begin{array}{l}\text { Case } d_{1}=d_{2} \\
\text { Case } d_{1}<d_{2} \\
\text { Case } d_{1}>d_{2}\end{array}$ & $\begin{array}{c}1.25 \mu \mathrm{m} \\
1 \mu \mathrm{m} \\
1.5 \mu \mathrm{m}\end{array}$ & $\begin{array}{c}1.25 \mu \mathrm{m} \\
1.5 \mu \mathrm{m} \\
1 \mu \mathrm{m}\end{array}$ \\
\hline
\end{tabular}

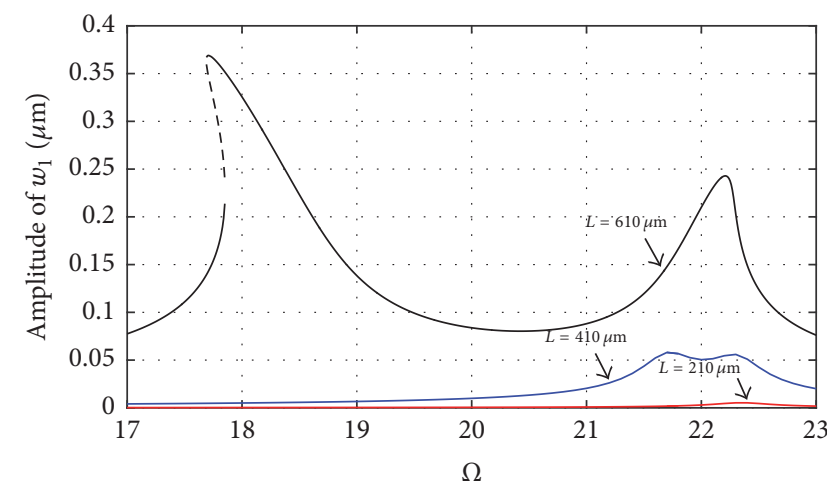

(a)

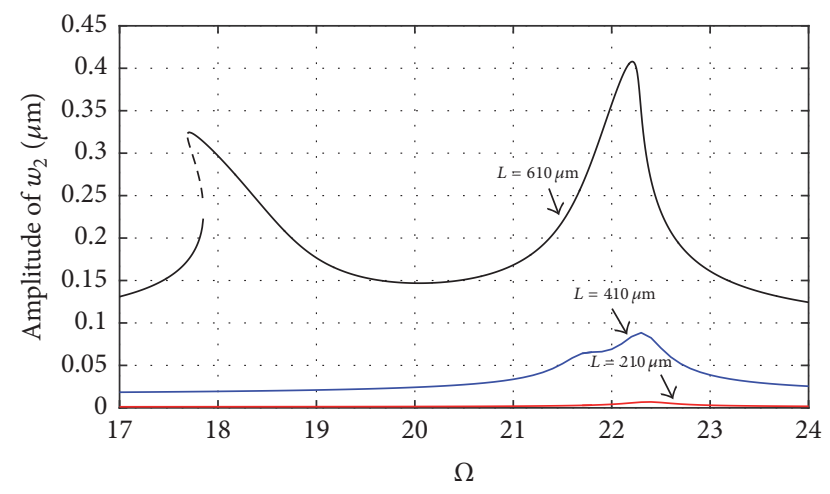

(b)

FIGURE 6: Effect of the microbeam length $(L)$ on the frequency responses of the (a) lower microbeam and (b) upper microbeam for $V_{\mathrm{DC}}=2$ Volts and $V_{\mathrm{AC}}=0.1$ Volts.

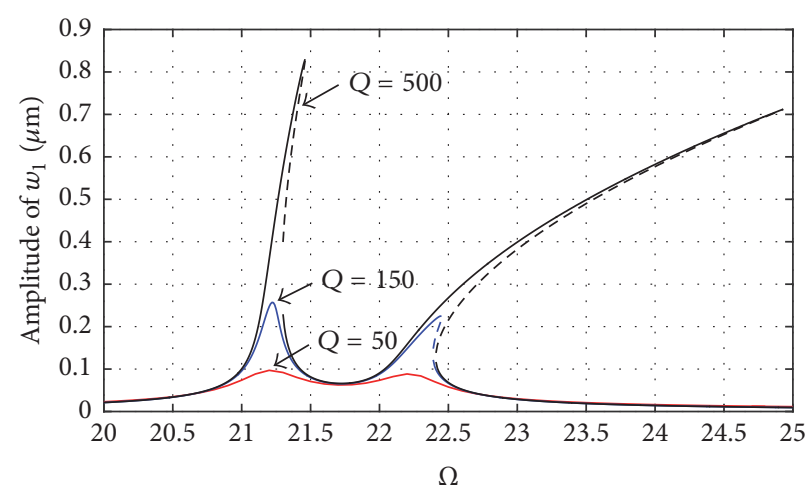

(a)

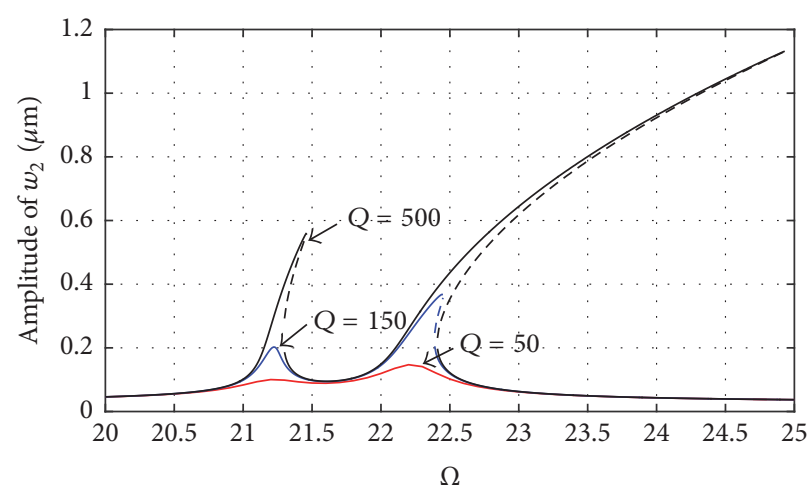

(b)

Figure 7: Effect of the quality factor $(Q)$ on the frequency responses of the (a) lower microbeam and (b) upper microbeam for $V_{\mathrm{DC}}=10$ Volts and $V_{\mathrm{AC}}=0.5$ Volts.

natural frequencies. However, the quality factor is shown to maximize the amplitude and with a high quality factor the nonlinearity becomes prominent (see hardening behavior in both figures).

6.3. Effect of the Applied DC Static Load $\left(V_{\mathrm{DC}}\right)$. The effects of the applied DC voltage on the amplitude are shown in Figure 8 . Three different voltages were selected, which are 5,10 , and 15 Volts. At a low DC voltage, the fundamental frequency is not affected by the applied voltage, so it is very close to its consecutive one. However, an increase in the value of the DC voltage causes the fundamental frequency to shift from its initial position, and hence the system gets closer and closer to the pull-in instability. Since the term of the applied AC voltage is small there is no presence for the nonlinearity. So, we would like to investigate the nonlinearity effect for the applied DC voltage at a high AC voltage. As it can be shown from Figure 9, by increasing the value of the applied AC voltage to 5 Volts, the hardening-type nonlinearity appears at high values of the $\mathrm{DC}$ voltage. This indicates that the mid-plane stretching term is significant, since it is responsible for this nonlinearity.

6.4. Effect of the Applied AC Harmonic Load $\left(V_{\mathrm{AC}}\right)$. Figure 10 shows the effect of the applied AC voltage on the amplitude. For this investigation, three different AC voltages were selected which are 5, 10, and 15 Volts. Unlike the DC 


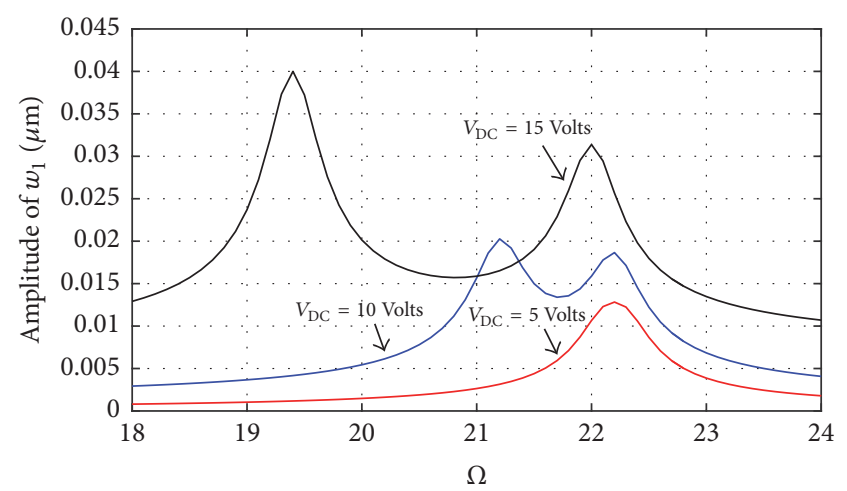

(a)

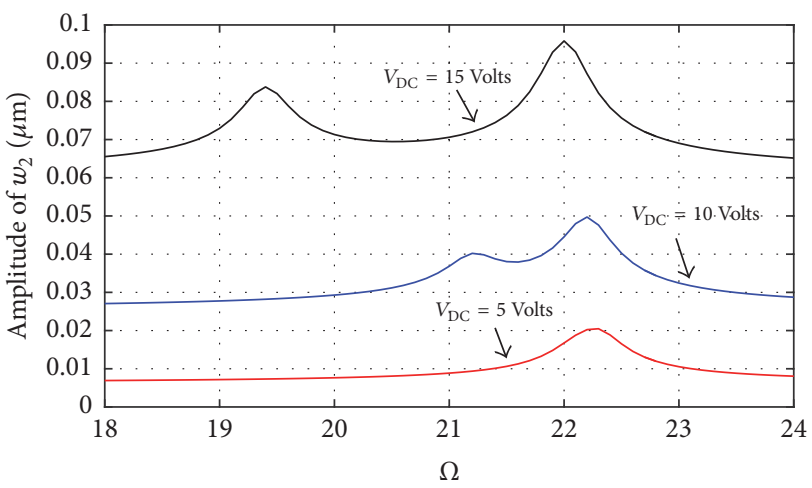

(b)

FIGURE 8: Effect of the applied DC voltage $\left(V_{\mathrm{DC}}\right)$ on the frequency responses of the (a) lower microbeam and (b) upper microbeam for $V_{\mathrm{AC}}=$ 0.1 Volts.

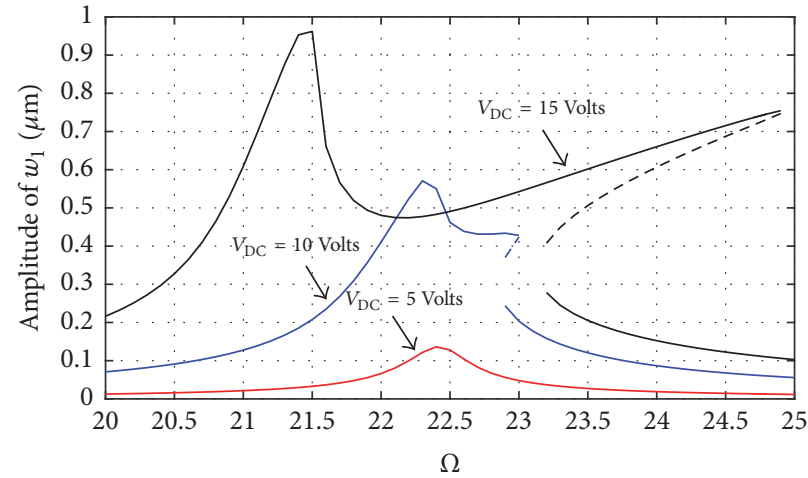

(a)

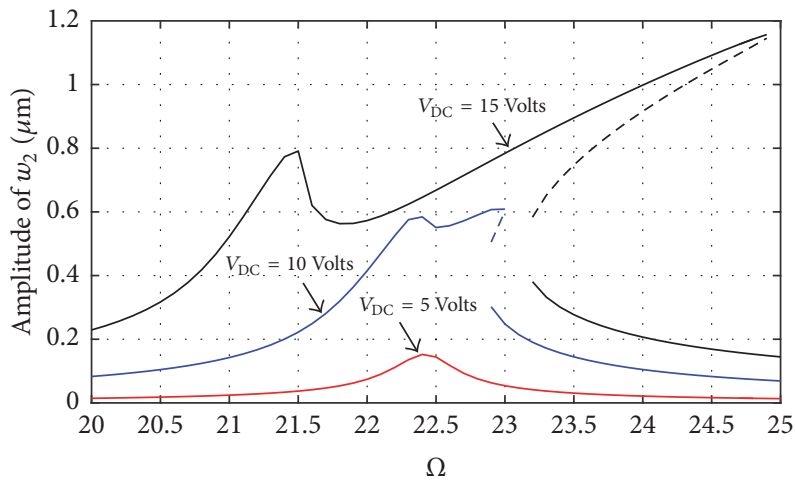

(b)

Figure 9: Effect of the applied DC voltage $\left(V_{\mathrm{DC}}\right)$ on the frequency responses of the (a) lower microbeam and (b) upper microbeam for $V_{\mathrm{AC}}=$ 5 Volts.

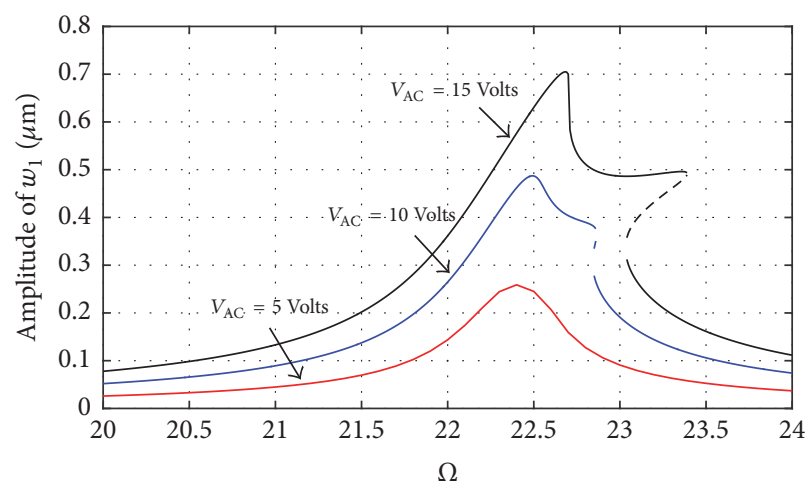

(a)

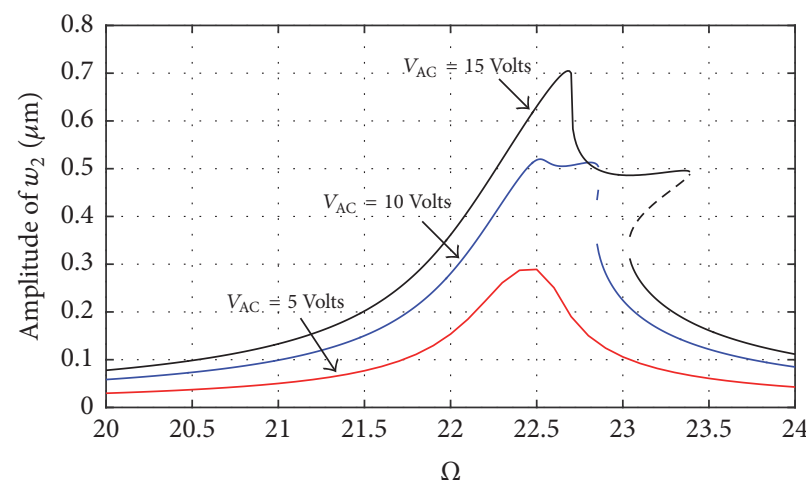

(b)

FIGURE 10: Effect of the applied AC voltage $\left(V_{\mathrm{AC}}\right)$ on the frequency responses of the (a) lower microbeam and $(\mathrm{b})$ upper microbeam for $V_{\mathrm{DC}}=$ 2 Volts.

voltage, the applied AC voltage has no effect on the natural frequencies, which is predictable since the dynamic loading has no effect on the natural frequencies. As shown in the figures, increasing the AC voltage increases the amplitude as well as producing a hardening type of nonlinearity.

\section{Conclusion}

An investigation into the nonlinear dynamics of a multilayers based MEMS resonator made of clamped-clamped microbeams under electrostatic actuation was conducted. A 
nonlinear Euler-Bernoulli beam model was utilized accounting for both geometric (mid-plane stretching) and actuation nonlinearities. Two numerical methods were used to discretize the continuous beam model, which are perturbation analysis and Galerkin based reduced-order modeling (ROM). The results showed that the outcomes of the two methods are in good agreement. Although the ROM is more accurate in obtaining the behavior, it cannot capture all of the solution's branches, where perturbation can be accomplished principally when the nonlinearity is becoming nontrivial.

The influences of different geometrical and design parameters were examined. Based on the parametric study, the effects of the resonator parameters selection were shown to be significant on the MEMS multilayers resonator dynamical profiles. For example, increasing the resonator length $(L)$ revealed a significant softening nonlinearity. The resonator quality factor $(Q)$ decrease showed a huge effect in maximizing the resonator dynamical response. Regarding the electric force actuating loads, increasing the DC voltage $\left(V_{\mathrm{DC}}\right)$ indicated a shift in the MEMS multilayers resonator resonant peaks away from each other, while producing a hardeningtype nonlinearity for higher $\mathrm{AC}$ voltages. Furthermore, assume that higher $\mathrm{AC}$ load $\left(V_{\mathrm{AC}}\right)$ produced more developed hardening behavior of the multilayers MEMS resonator. Consequently, interesting dynamical features can be achieved by controlling these resonator parameters, which may be useful in numerous MEMS resonating/sensing applications.

\section{Competing Interests}

The authors declare no potential conflict of interests with respect to the research, authorship, and/or publication of this article.

\section{Acknowledgments}

The authors greatly appreciate the support received from King Fahd University of Petroleum and Minerals (KFUPM) through its Deanship of Scientific Research (DSR).

\section{References}

[1] M. I. Younis, "Introduction to Nonlinear Dynamics," in MEMS Linear and Nonlinear Statics and Dynamics, vol. 20 of Microsystems, pp. 155-249, Springer US, Boston, Mass, USA, 2011.

[2] M. Gad-el-Hak, The MEMS Handbook, CRC Press, 2001.

[3] M. Tanaka, "An industrial and applied review of new MEMS devices features," Microelectronic Engineering, vol. 84, no. 5-8, pp. 1341-1344, 2007.

[4] M. H. Ghayesh, M. Amabili, and H. Farokhi, "Three-dimensional nonlinear size-dependent behaviour of Timoshenko microbeams," International Journal of Engineering Science, vol. 71, pp. 1-14, 2013.

[5] E. M. Miandoab, A. Yousefi-Koma, H. N. Pishkenari, and F. Tajaddodianfar, "Study of nonlinear dynamics and chaos in MEMS/NEMS resonators," Communications in Nonlinear Science and Numerical Simulation, vol. 22, no. 1-3, pp. 611-622, 2015.
[6] M. I. Younis and A. H. Nayfeh, "A study of the nonlinear response of a resonant microbeam to an electric actuation," Nonlinear Dynamics, vol. 31, no. 1, pp. 91-117, 2003.

[7] S. Dou and J. S. Jensen, "Optimization of nonlinear structural resonance using the incremental harmonic balance method," Journal of Sound and Vibration, vol. 334, pp. 239-254, 2015.

[8] H. M. Ouakad and M. I. Younis, "The dynamic behavior of MEMS arch resonators actuated electrically," International Journal of Non-Linear Mechanics, vol. 45, no. 7, pp. 704-713, 2010.

[9] E. S. Hung and S. D. Senturia, "Generating efficient dynamical models for microelectromechanical systems from a few finiteelement simulation runs," Journal of Microelectromechanical Systems, vol. 8, no. 3, pp. 280-289, 1999.

[10] F. Najar, S. Choura, E. M. Abdel-Rahman, S. El-Borgi, and A. Nayfeh, "Dynamic analysis of variable-geometry electrostatic microactuators," Journal of Micromechanics and Microengineering, vol. 16, no. 11, pp. 2449-2457, 2006.

[11] E. M. Abdel-Rahman, M. I. Younis, and A. H. Nayfeh, "Characterization of the mechanical behavior of an electrically actuated microbeam," Journal of Micromechanics and Microengineering, vol. 12, no. 6, pp. 759-766, 2002.

[12] H. M. Ouakad and M. I. Younis, "Modeling and simulations of collapse instabilities of microbeams due to capillary forces," in Proceedings of the ASME International Mechanical Engineering Congress and Exposition (IMECE '08), pp. 187-195, Boston, Mass, USA, November 2008.

[13] A. M. Bouchaala and M. I. Younis, "A model of electrostatically actuated MEMS and carbon nanotubes resonators for biological mass detection," in Design and Modeling of Mechanical Systems-II, pp. 501-512, Springer, 2015.

[14] D. I. Caruntu and I. Martinez, "Reduced order model of parametric resonance of electrostatically actuated MEMS cantilever resonators," International Journal of Non-Linear Mechanics, vol. 66, pp. 28-32, 2014.

[15] M. I. Younis, E. M. Abdel-Rahman, and A. Nayfeh, "A reducedorder model for electrically actuated microbeam-based MEMS," Journal of Microelectromechanical Systems, vol. 12, no. 5, pp. 672-680, 2003.

[16] A. H. Nayfeh, M. I. Younis, and E. M. Abdel-Rahman, "Reduced-order models for MEMS applications," Nonlinear Dynamics. An International Journal of Nonlinear Dynamics and Chaos in Engineering Systems, vol. 41, no. 1-3, pp. 211-236, 2005.

[17] G. C. Turner and M. K. Andrews, "Frequency stabilisation of electrostatic oscillators," in Proceedings of the 8th International Conference on Solid-State Sensors and Actuators, Eurosensors IX, Transducers, pp. 624-626, Stockholm, Sweden, June 1995.

[18] A. Alofi, H. Ouakad, and M. Tausiff, "Structural behavior of a multi-layer based microbeam actuator," Actuators, vol. 5, no. 3, p. 22, 2016.

[19] J. P. Chaffey and M. Austin, "Analytical modeling of the electromechanical coupling of cantilever beams," in Smart Structures, Devices, and Systems, vol. 4935 of Proceedings of SPIE, pp. 86-93, Melbourne, Australia, December 2002.

[20] S. Afrang and E. Abbaspour-Sani, "A low voltage MEMS structure for RF capacitive switches," Progress in Electromagnetics Research, vol. 65, pp. 157-167, 2006.

[21] H. Samaali, F. Najar, S. Choura, A. H. Nayfeh, and M. Masmoudi, "A double microbeam MEMS ohmic switch for RFapplications with low actuation voltage," Nonlinear Dynamics, vol. 63 , no. 4, pp. 719-734, 2011. 
[22] H. M. Ouakad, M. A. Hawwa, and H. M. Al-Qahtani, "Modeling the electrostatic deflection of a MEMS multilayers based actuator," Mathematical Problems in Engineering, vol. 2013, Article ID 959232, 6 pages, 2013.

[23] H. Samaali, F. Najar, and S. Choura, "Dynamic study of a capacitive MEMS switch with double clamped-clamped microbeams," Shock and Vibration, vol. 2014, Article ID 807489, 7 pages, 2014.

[24] A. H. Nayfeh, Introduction to Perturbation Techniques, John Wiley \& Sons, 2011. 


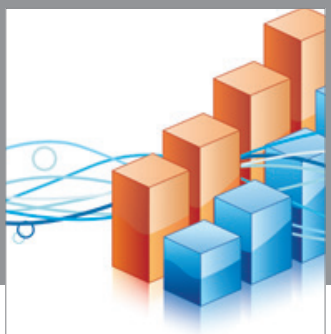

Advances in

Operations Research

vatem alat4

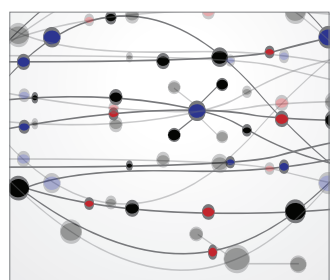

\section{The Scientific} World Journal
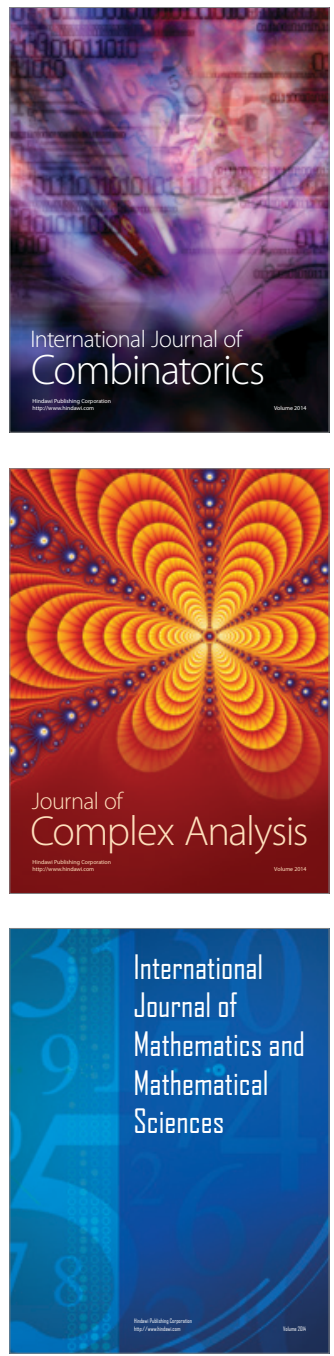
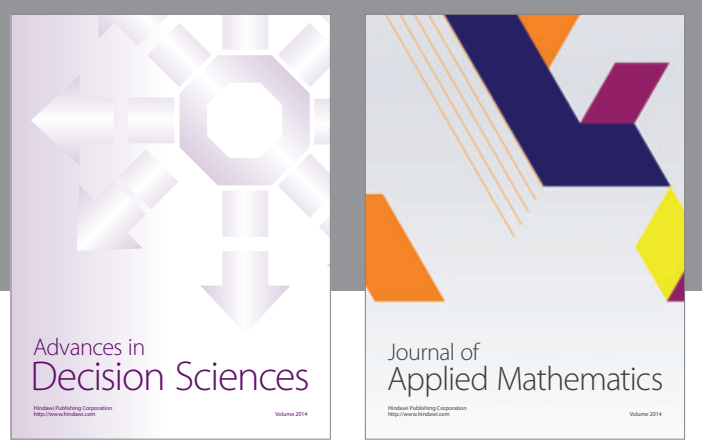

Algebra

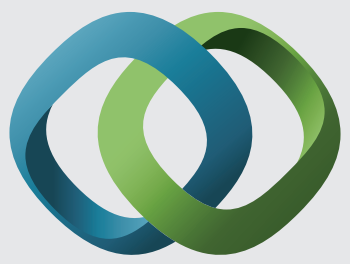

\section{Hindawi}

Submit your manuscripts at

https://www.hindawi.com
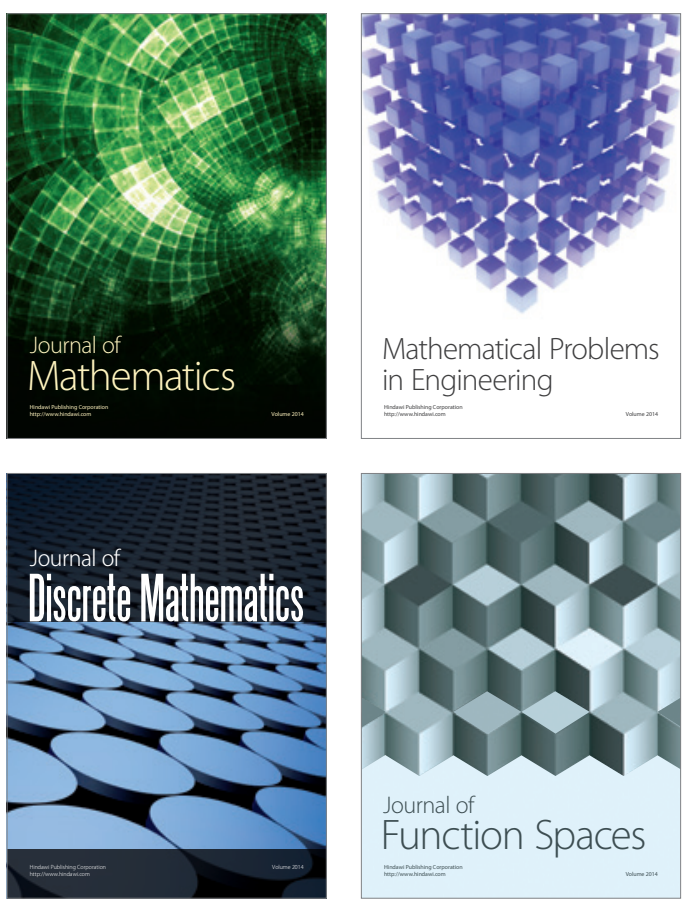

Mathematical Problems in Engineering
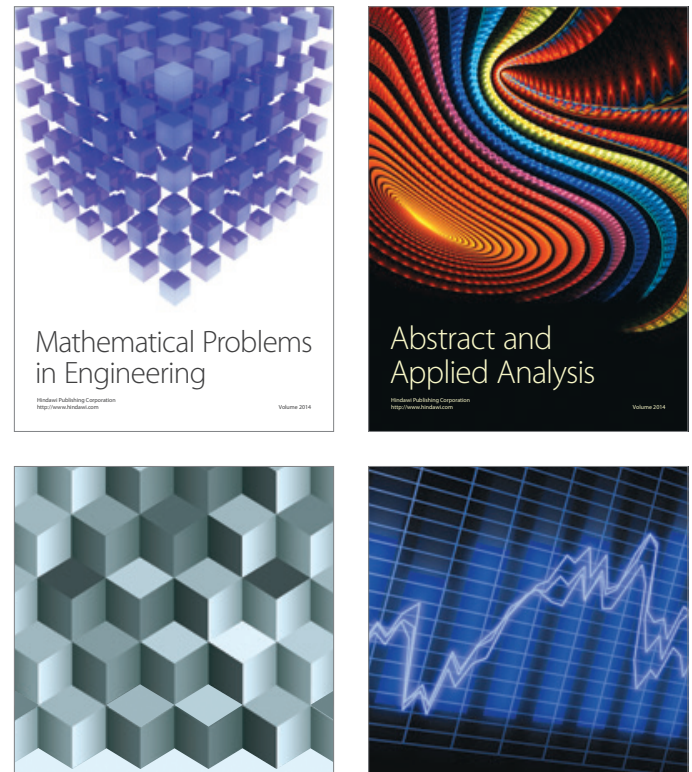

Journal of

Function Spaces

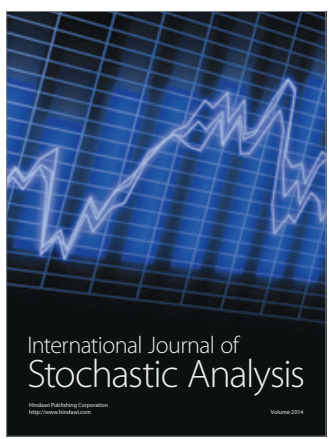

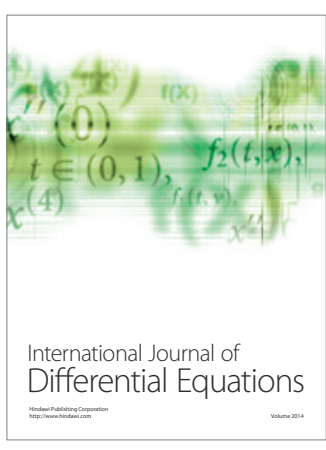
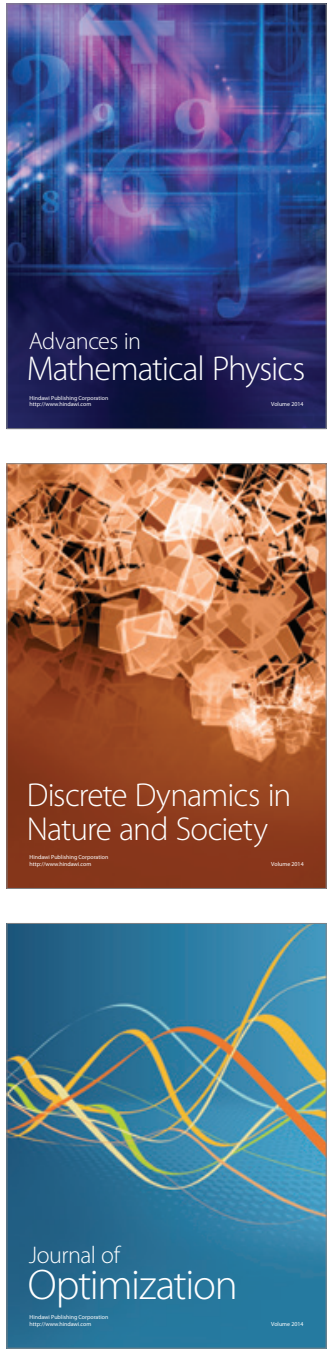